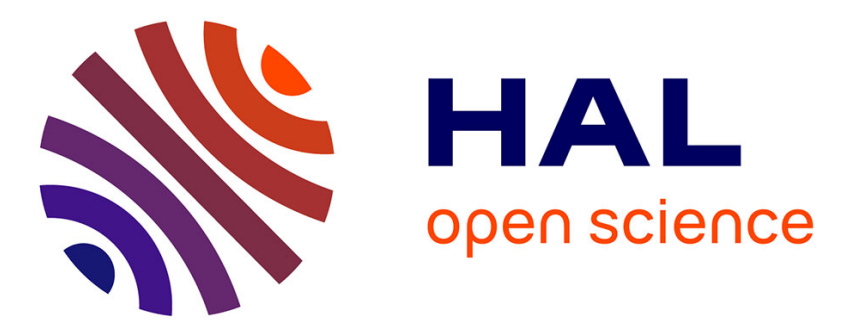

\title{
A comparative study on few-layer graphene production by exfoliation of different starting materials in a low boiling point solvent
}

\author{
Yasemin Çelik, Emmanuel Flahaut, Ender Suvaci
}

\section{- To cite this version:}

Yasemin Çelik, Emmanuel Flahaut, Ender Suvaci. A comparative study on few-layer graphene production by exfoliation of different starting materials in a low boiling point solvent. FlatChem, 2017, vol. 1, pp. 74-88. 10.1016/j.flatc.2016.12.002 . hal-01537861

\author{
HAL Id: hal-01537861 \\ https://hal.science/hal-01537861
}

Submitted on 13 Jun 2017

HAL is a multi-disciplinary open access archive for the deposit and dissemination of scientific research documents, whether they are published or not. The documents may come from teaching and research institutions in France or abroad, or from public or private research centers.
L'archive ouverte pluridisciplinaire HAL, est destinée au dépôt et à la diffusion de documents scientifiques de niveau recherche, publiés ou non, émanant des établissements d'enseignement et de recherche français ou étrangers, des laboratoires publics ou privés. 


\section{Open Archive TOULOUSE Archive Ouverte (OATAO)}

OATAO is an open access repository that collects the work of Toulouse researchers and makes it freely available over the web where possible.

This is an author-deposited version published in : http://oatao.univ-toulouse.fr/ Eprints ID : 17897

To link to this article : DOI:10.1016/j.flatc.2016.12.002

URL : http://dx.doi.org/10.1016/j.flatc.2016.12.002

To cite this version : Çelik, Yasemin and Flahaut, Emmanuel and Suvac1, Ender A comparative study on few-layer graphene production by exfoliation of different starting materials in a low boiling point solvent. (2017) FlatChem, vol. 1. pp. 74-88. ISSN 2452-2627

Any correspondence concerning this service should be sent to the repository administrator: staff-oatao@listes-diff.inp-toulouse.fr 


\title{
A comparative study on few-layer graphene production by exfoliation of different starting materials in a low boiling point solvent
}

\author{
Yasemin Çelik $^{\mathrm{a}, \mathrm{b}, *}$, Emmanuel Flahaut $^{\mathrm{b}}$, Ender Suvacı $^{\mathrm{a}}$ \\ ${ }^{a}$ Anadolu University, Department of Materials Science and Engineering, 26480 Eskişehir, Turkey

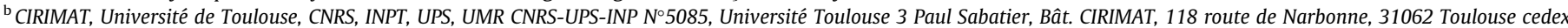 \\ 9, France
}

\begin{abstract}
A B S T R A C T
Three different graphite-based powders (expandable graphite and two different nano-graphite powders) were investigated as starting materials for an effective liquid phase exfoliation process in isopropyl alcohol (IPA). The prepared dispersions were analyzed and compared in terms of their graphene concentration, stability, number of graphene layers and quality, as well as the electrical conductivity of the prepared graphene-based materials. Good quality graphene dispersions $\left(I_{\mathrm{D}} / I_{\mathrm{G}}<0.3\right)$ with a relatively high concentration $(\sim 1.1 \mathrm{mg} / \mathrm{ml})$ were prepared in IPA within 90 min sonication time by utilizing a high specific surface area $\left(\sim 175 \mathrm{~m}^{2} / \mathrm{g}\right)$ nano-graphite powder derived from natural graphite. Transmission electron microscope analyses of this sample revealed mostly folded and scrolled few layer graphene (FLG) sheets ( $<5$ layers) entangled each other. The electrical conductivity of the thin film prepared from this dispersion was $\sim 15$ and $86 \mathrm{~S} / \mathrm{m}$, before and after annealing, respectively. FLG prepared from expanded graphite, obtained by thermal treatment of expandable graphite, exhibited both much higher quality $\left(I_{\mathrm{D}} /\right.$ $\left.I_{\mathrm{G}}<0.09\right)$ and electrical conductivity ( $\sim 2104$ and $19,200 \mathrm{~S} / \mathrm{m}$ before and after annealing, respectively) when dispersed in IPA for $90 \mathrm{~min}$. However, the graphene-based material concentration of the prepared dispersion was relatively low $(\sim 0.06 \mathrm{mg} / \mathrm{ml})$.
\end{abstract}

\section{Keywords:}

Exfoliation

Expanded graphite

Nano-graphite

Few-layer graphene sheets

\section{Introduction}

Graphite is an inexpensive and readily available precursor for production of graphene-based materials (single layer, few-layer or multi-layer graphene sheets, graphene oxide, reducedgraphene oxide, etc.). The main challenge of graphene production from graphite is to overcome van der Walls forces which hold graphene sheets together. Liquid phase exfoliation is a promising method to exfoliate graphite into graphene sheets, dispersed as a colloidal suspension in a solvent with or without surfactant. This process eliminates the need of using a substrate. Since there is no graphene transfer step, the produced graphene-based materials can be readily incorporated into other materials such as ceramics or polymers to form composites. The critical point in liquid phase exfoliation is to be able to increase graphene concentration as much as possible while maintaining the quality of the flakes and keeping their lateral size large enough.

\footnotetext{
* Corresponding author at: Anadolu University, Department of Materials Science and Engineering, 26480 Eskişehir, Turkey.

E-mail address: ybozkaya@anadolu.edu.tr (Y. Çelik).
}

Comprehensive reviews were published on exfoliation of graphite powders into single- and few-layer graphene sheets in various liquids, including organic solvents, ionic liquids, and water/surfactant solutions [1-4]. The qualities, yields and electrical properties of exfoliated graphene samples are also reviewed in these papers. Zhong et al. [5] have recently reviewed wet chemical graphite exfoliation routes highlighting their progress and challenges in terms of graphene commercialization. There have been several attempts to produce graphene-based materials at a large-scale. Exfoliation of graphite in aqueous solutions with aid of surfactants yielded graphene concentrations of mostly $<1 \mathrm{mg}$ / $\mathrm{ml}$ [6]. Concentration was further increased up to $15 \mathrm{mg} / \mathrm{ml}$ by continuous addition of surfactant throughout the sonication process [7]. Ager et al. [8] demonstrated complete exfoliation of up to $5 \mathrm{wt} \%$ graphene in water by using triblock copolymers and copolymeric nanolatexes based on a reactive ionic liquid acrylate surfactant for extended time periods of sonication. Ayán-Varela et al. [9] recently reported very high graphene concentrations (up to $\sim 50 \mathrm{mg} \mathrm{ml}^{-1}$ ) in aqueous dispersions by using the sodium salt of flavin mononucleotide biomolecule as a surfactant in an exfoliation process which was carried out in an ultrasonic bath for $5 \mathrm{~h}$. However, most surfactants are insulating compounds that should 
be removed from the system by an additional washing step for further applications; moreover, using relatively high amount of surfactants may bring additional cost [5]. Chlorosulphonic acid was also reported as a highly efficient solvent for graphene production with concentration values up to $2 \mathrm{mg} / \mathrm{ml}$ [10]; however, it is toxic, highly corrosive and reactive which prevents its use for further processing. Graphite can also be exfoliated into high-quality graphene sheets (with <5 layers) in 1-methyl-2-pyrrolidone (NMP, $\mathrm{C}_{5} \mathrm{H}_{9} \mathrm{NO}$ ) due to well matched surface energy between graphene and the solvent [11]. Therefore, it is one of the most widely preferred organic solvent for sonication assisted liquid-phase exfoliation of graphene from graphite. Initial studies of direct exfoliation of graphene in organic solvents resulted in graphene dispersions at very low concentrations of $0.01 \mathrm{mg} / \mathrm{ml}$ [11]. Khan et al. [12] showed that the concentration of graphene dispersed in NMP can be increased dramatically (up to $1.2 \mathrm{mg} / \mathrm{ml}$ ) by sonicating at low power for very long times $(\sim 460 \mathrm{~h})$. Recently, Khan et al. [13] increased graphene concentration up to $2 \mathrm{mg} / \mathrm{ml}$ by simple tip sonication of graphite for $6 \mathrm{~h}$ in NMP, followed by centrifugation. The authors further increased graphene concentration up to $63 \mathrm{mg} / \mathrm{ml}$ at a yield of $19 \%$ (percentage of graphite exfoliated as few-layer graphene) by re-dispersing the exfoliated material after centrifugation [13]. Catheline et al. [14] reported that graphite intercalation compound $\mathrm{KC}_{8}$ spontaneously dissolves in NMP, yielding solutions of negatively charged graphene layers with a concentration of $0.7 \mathrm{mg} / \mathrm{ml}$ after stirring overnight and centrifugation to remove insoluble material.

Although these studies have shown that NMP is an effective solvent to exfoliate graphite, its high boiling point $\left(\sim 204{ }^{\circ} \mathrm{C}\right.$ at $760 \mathrm{mmHg}$ ) makes it difficult to be completely removed from the system and the residual solvent can be detrimental for composites. Moreover, this may cause problems during flake deposition onto a substrate, since agglomeration tends to occur during slow solvent evaporation [15]. Hence, exfoliating graphite in a low boiling point solvent to achieve graphene-based dispersions with a concentration as high as possible would facilitate applicability of these materials into composites and deposition onto substrates. O'Neill et al. [15] demonstrated graphene concentrations of up to $0.5 \mathrm{mg} / \mathrm{ml}$ in isopropyl alcohol (IPA), whose boiling point is $82.5^{\circ} \mathrm{C}$, by a low power sonication of graphite for $48 \mathrm{~h}$ followed by a centrifugation at $500 \mathrm{rpm}$, showing that it is possible to produce graphene dispersions with a relatively high concentration in low boiling point solvents. However, long process times make the method impractical for realizing the potential applications. Therefore, this process needs to be improved so as to achieve a higher graphene concentration within a shorter time.

The choice of starting materials, as well as the use of an appropriate solvent, is critical for liquid phase exfoliation process. The most commonly used starting material for the production of graphene-based materials by liquid phase exfoliation is natural graphite. The number of studies which use expandable/expanded graphite and nano-graphite powders as precursors is limited $[14,16]$. Kozhemyakina et al. [17] have recently published a comprehensive study in which they investigated the dispersibility of several types of graphite with different morphological and structural characteristics in two high boiling point organic solvents (including NMP) and in one surfactant-water solution. The authors followed an uncommon way to prepare their dispersions: they stirred graphite in the corresponding solvent over a period of twodays. The carbon uptake of the graphite materials into solvents was determined by absorption measurements on the decanted part after leaving them for 1 day of sedimentation. It was observed that the dispersibility of graphite materials with small grain size is better than that of large grain sized graphite materials, and this depends not only on the nature of the graphite but also on the viscosity of the solvent used [17].
The objective of this study was to investigate three different graphite materials (expandable graphite, nano-graphite powder derived from natural graphite and nano-graphite powder derived from synthetic graphite) as starting powders for liquid phase exfoliation in a low boiling point solvent (IPA) in order to produce good quality graphene-based materials at a large-scale within relatively short sonication times ( $<120 \mathrm{~min}$ ). The prepared graphene-based dispersions were characterized and compared in terms of their concentration and stability, and number of layers, quality and electrical conductivity of the obtained graphene-based materials.

\section{Methods}

\section{Starting materials and exfoliation process}

Three different graphite-based materials, expandable graphite, surface enhanced flake graphite (SEFG) and primary artificial (PA) were used as starting powders for the exfoliation studies. The expandable graphite (Expansion ratio: 307, Grade 3772), SEFG (Grade 3725) and the PA (Grade TC307) were kindly provided by Asbury Carbons Inc. The SEFG, which was derived from natural flake graphite and the PA, which was derived from synthetic graphite are nano-graphite powders, the specific surface area (SSA) of which are 175 and $350 \mathrm{~m}^{2} / \mathrm{g}$, respectively. These two powders were used as-received for further exfoliation studies, while expandable graphite was subjected to an abrupt heating in a preheated furnace at $900{ }^{\circ} \mathrm{C}$ for $2 \mathrm{~min}$ in air to prepare expanded graphite (EG) prior to exfoliation. Before thermal expansion, the expandable graphite was first dried in a vacuum oven and then a small amount of dried powder was put at the bottom of an alumina crucible and placed in the furnace.

The SEFG and PA powders were dispersed in IPA (MerckEmsure) and also in NMP (Merck-Emplura) at an initial concentration of $3 \mathrm{mg} / \mathrm{ml}$ by sonicating in an ultrasonic bath (Kudos, $35 \mathrm{kHz}$, $100 \mathrm{~W}$ ) for $10-120 \mathrm{~min}$. The EG was dispersed in IPA at an initial concentration of $0.2 \mathrm{mg} / \mathrm{ml}$ by bath sonication $(100 \mathrm{~W})$, as well as using tip sonication (Cole Parmer, CP750, $20 \mathrm{kHz}$, running at $250 \mathrm{~W}$ ) in order to observe the effect of sonication power on graphene concentration. Large graphitic flakes were removed from the dispersions of the SEFG and PA by a subsequent centrifugation at $500 \mathrm{rpm}$ for $45 \mathrm{~min}$ unless otherwise stated. The dispersions prepared from the EG powder were centrifuged at $900 \mathrm{rpm}$ for 45 min. After centrifugation, the top $\sim 90 \%$ of the supernatant was removed by pipetting for further use. Prepared dispersions were denoted as 'Precursor-Solvent-Sonication time'. This is valid for the dispersions which were prepared in ultrasonic bath and centrifuged at $500 \mathrm{rpm}$ for $45 \mathrm{~min}$. For the tip sonicated samples, the abbreviation 'TS' was added at the end; likewise, for the samples centrifuged at $900 \mathrm{rpm}$ for $45 \mathrm{~min}$, the term of ' $900 \mathrm{rpm}$ ' was added at the end.

\section{Characterization of the starting powders and the exfoliated materials}

Field emission gun-scanning electron microscopy (FEG-SEM) analyses of the precursor powders and the dispersed graphene/graphite sheets were performed using Zeiss Supra 50VP. FEGSEM samples were prepared by dropping some dispersion onto a Si substrate with an oxide layer thickness of $300 \mathrm{~nm}$ and subsequently drying in an oven (at $\sim 80^{\circ} \mathrm{C}$ in air). Lateral size distribution of exfoliated graphene/graphite flakes and zeta potential of dispersions were measured by dynamic light scattering (DLS) and electrophoretic light scattering (ELS), respectively, using Malvern Zetasizer Nano ZS system with a $633 \mathrm{~nm}$ laser. The stability of the prepared graphene-based dispersions was investigated by observing their sedimentation behavior. The sedimentation study 
was performed by allowing the dispersions to sediment for 8 weeks and measuring the optical absorbance (at $660 \mathrm{~nm}$ ) of the supernatant at 1-3 weeks of intervals. UV-VIS absorbance spectra of the graphene-based dispersions and the pure solvents were recorded in the $200-800 \mathrm{~nm}$ wavelength range using Varian Cary100Bio spectrometer with $1 \mathrm{~cm}$ cuvettes. The concentration of graphene remaining after centrifugation was determined by measuring the absorbance at $660 \mathrm{~nm}$. The absorbance value was transformed into concentration using Beer-Lambert Law [18], Eq. (1):

$\mathrm{A}=\alpha \times l \times \mathrm{C}$

Accordingly, absorbance $(A)$ of the suspension is proportional to the concentration (C), the cell length (l) and the absorption coefficient $(\alpha)$, which was taken as $2460 \mathrm{ml} \mathrm{mg}^{-1} \mathrm{~m}^{-1}$ [11]. For comparison with the literature, ' $\alpha$ ' was also determined experimentally for one of the dispersions (SEFG-IPA-90 min). For this purpose, large volume of dispersions $(\sim 350-400 \mathrm{ml})$ were prepared and filtered through a pre-weighted PVDF membrane by vacuum filtration using a Buchner Funnel. After filtration, the membranes were dried in an oven at $\sim 80^{\circ} \mathrm{C}$ overnight. The dried membranes were weighed again and the mass of the graphitic material on the membrane was determined. Finally, the absorption coefficient $(\alpha)$ was determined using the Beer-Lambert Law. Accordingly, for the SEFG-IPA90 min dispersion ' $\alpha$ ' was calculated as $\sim 2468 \mathrm{ml} \mathrm{mg}^{-1} \mathrm{~m}^{-1}$ (the average of three measurements). This value is almost the same as the one estimated by Hernandez et al. [11] as $2460 \mathrm{ml} \mathrm{mg}^{-1} \mathrm{~m}^{-1}$, although the same group was later determined the ' $\alpha$ ' as $3620 \mathrm{ml} \mathrm{mg}^{-1} \mathrm{~m}^{-1}$ for these type of systems [12].

Micro-Raman analysis and electrical characterization of the samples were performed on thin films prepared from the dispersions. Sheet resistance measurements were carried out by fourpoint probe technique using Lucas Labs Pro4 Resistivity System. The conductivity values of the thin films were calculated from the measured sheet resistances using Eq. (2)

$\sigma_{\mathrm{el}}=\mathrm{R}_{\mathrm{s}}^{-1} \times \mathrm{t}^{-1}$

where $\sigma_{\mathrm{el}}$ is the electrical conductivity; $R_{S}$ is the sheet resistance and $t$ is the thickness of the film.

Micro-Raman analyses of the starting materials and the exfoliated samples were performed by Renishaw Invia spectrometer using $532 \mathrm{~nm}$ laser $(2.33 \mathrm{eV})$ excitation and $100 \times$ objective lens. The laser power was kept below $1 \mathrm{~mW}$ in order to prevent sample damage. 50 spectra were recorded (each one at a different location) for each sample to create statistical histogram of the $I_{\mathrm{D}} / I_{\mathrm{G}}$ ratio.

Transmission Electron Microscopy (TEM) analyses (both low magnification and high resolution) were performed with a Jeol JEM 2100F. TEM samples were prepared by drop casting the graphene-based dispersions onto holey carbon coated copper grids (200 mesh). In order to investigate the effect of high temperature annealing on the quality of graphene-based materials, one of the exfoliated samples (SEFG-IPA-90 min) was annealed at $2600^{\circ} \mathrm{C}$ in $\mathrm{Ar}$ atmosphere for $4 \mathrm{~h}$, and further characterized by Raman spectrometer and TEM.

\section{Thin film preparation}

Thin films were prepared by vacuum filtration of the graphenebased dispersions through porous alumina membranes (Whatman Anodisc $47 \mathrm{~mm}, 0.02 \mu \mathrm{m}$ pore size). After vacuum filtration, the thin films prepared from IPA-based dispersions were dried in air at room temperature, while the thin films prepared from NMP-based dispersions were dried in vacuum oven at $25 \mathrm{hPa}$ and $50{ }^{\circ} \mathrm{C}$ for overnight. The thickness of the films was calculated using Eq. (3):

$\frac{\pi D^{2}}{4} t \rho=V C$ where $D$ is the diameter of the film $(\sim 4 \mathrm{~cm}) ; t$ is the thickness of the film; $\rho$ is the density of the graphene-based material (assumed as $2.2 \mathrm{~g} / \mathrm{cm}^{3}$ - the density of bulk graphite); $V$ is the volume of the dispersion and $C$ is the concentration of graphene in dispersion $(\mathrm{mg} /$ ml) [19]. This equation was previously used by Yu et al. [19] for calculation of the thickness of carbon nanotube films; here it was adapted for graphene-based films. Accordingly, it was found that the thickness values of the graphene-based thin films range from 43 to $86 \mathrm{~nm}$ depending on the volume and the concentration of the dispersions used.

\section{Results and discussion}

\section{Exfoliation of the starting materials}

Expandable graphite is a form of intercalated graphite, i.e., an intercalant material is inserted between the graphene layers of a graphite crystal. The expandable graphite material used in the present study (supplied from Asbury Carbon Inc.) is manufactured by treating highly crystalline natural flake graphite with a mixture of sulfuric acid and certain other oxidizing agents like potassium permanganate and/or nitric acid which aid in catalysis of the sulfate intercalation. FEG-SEM micrographs of the as-received expandable graphite reveal the lamellar structure of the flakes with an average size of $\sim 450 \mu \mathrm{m}$ and a thickness of mostly $<50 \mu \mathrm{m}$ (Fig. 1 (a) and (b)). There are also small amount of thicker flakes up to $180 \mu \mathrm{m}$ in thickness. Abrupt heating of expandable graphite at $900{ }^{\circ} \mathrm{C}$ for 2 min caused expansion agent to gasify, pushing adjacent graphene layers apart, forming expanded graphite 'EG'. The expansion temperature was determined according to thermogravimetric analysis (performed in air up to $1000{ }^{\circ} \mathrm{C}$ with a heating rate of $10^{\circ} \mathrm{C} / \mathrm{min}$ ) result of expandable graphite, which exhibited twostep weight loss. The sample started to degrade at $\sim 170{ }^{\circ} \mathrm{C}$ accompanied with a mass drop due to release of gaseous intercalation species from the expandable graphite interlayers, as in agreement with Chiang and Hsu [20] who reported that expandable graphite starts to decompose at $170-250{ }^{\circ} \mathrm{C}$. Another weight loss was observed at $\sim 600-1000{ }^{\circ} \mathrm{C}$ due to combustion of graphite to carbon dioxide [21]. Accordingly, the expansion temperature was determined as $900{ }^{\circ} \mathrm{C}$ and the degree of expansion was investigated depending on expansion time by varying it from $10 \mathrm{~s}$ to $2 \mathrm{~min}$. Heating of the expandable graphite at $900^{\circ} \mathrm{C}$ for $10 \mathrm{~s}$ did not lead to considerable volume increase due to remaining unexpanded flakes and incomplete gas release which caused formation of bubbles on the surface of layers (Fig. 1(c)). Fig. 1 (d) and (e) show characteristic loose and porous worm-like (vermicular) structure of expanded graphite, where many large pores formed due to separation of graphite layers and still attaching together at their edges in some parts by weak van der Waals forces. Expanded graphite exhibited smaller and less amount of pores when heated for $2 \mathrm{~min}$ in comparison to heating for $1 \mathrm{~min}$, indicating a better expansion. Exfoliation of EG in IPA by tip sonication for $90 \mathrm{~min}$ followed by centrifugation resulted in thin, transparent flakes with lateral sizes of $\sim 1-5 \mu \mathrm{m}$ (Fig. 1(f)).

The dispersibility of EG in IPA was examined using the relationship between the absorbance and the concentration (Beer-Lambert Law) [18]. UV-VIS absorption spectrum of the EG-IPA-90 min dispersion was flat and featureless [11] except the peak at $\sim 264 \mathrm{~nm}$ which is characteristic to graphene and can be attributed to the $\pi \rightarrow \pi^{*}$ transitions of aromatic $\mathrm{C}=\mathrm{C}$ bonds [22]. The concentration of graphene-based material remaining after centrifugation was determined by measuring the absorbance of the dispersions at $660 \mathrm{~nm}$. The concentration showed an increasing trend with increasing sonication time up to $90 \mathrm{~min}$ (Fig. 2). In the case of bath sonication, 90 and 120 min sonication led to similar concentration 

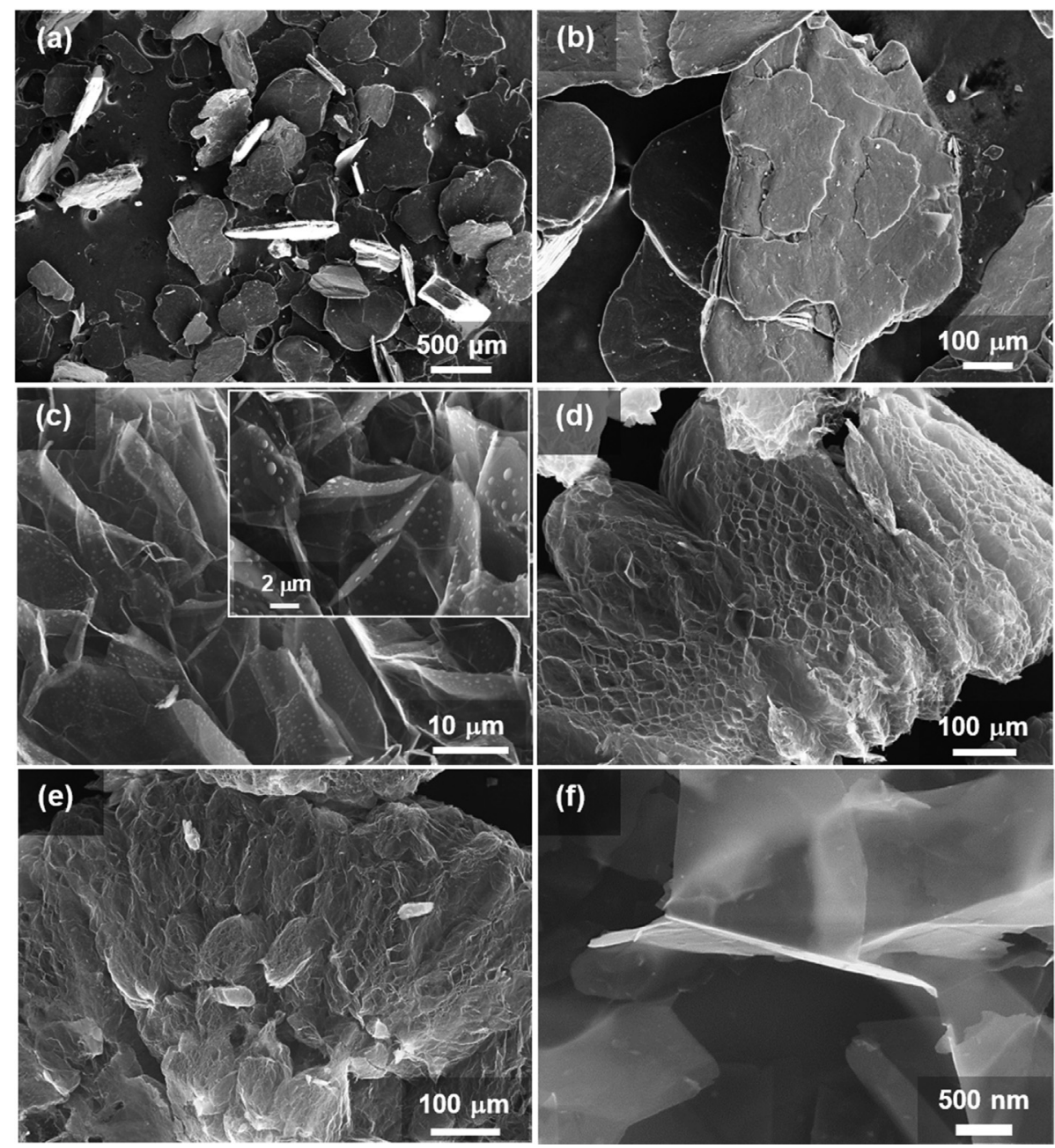

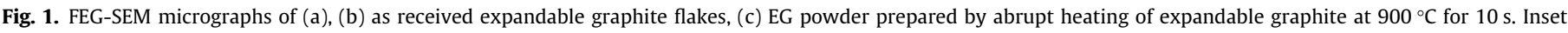

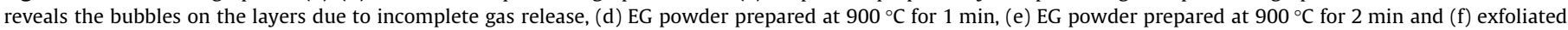
material obtained by tip sonication of EG in IPA for 90 min followed by centrifugation (EG-IPA-90 min-TS).

values, while 120 min tip sonication resulted in a slightly higher concentration in comparison to $90 \mathrm{~min}$. Although tip sonication provided a higher concentration than that of sonic bath (e.g., 0.06 and $0.04 \mathrm{mg} / \mathrm{ml}$ for tip and bath sonication for $120 \mathrm{~min}$, respectively), these values are much below the requirements for the large-scale production of graphene. Dhakate et al. [16] achieved a concentration of $0.05 \mathrm{mg} / \mathrm{ml}$ by exfoliation of EG via ultrasonication and centrifugation in DMF. The authors then applied re-intercalation and re-exfoliation of the EG in order to obtain monolayer and bilayer graphene, the yield of which was reported as $\sim 4-5 \mathrm{wt} \%$ (mass of monolayer or bilayer/starting EG mass) [16].

SEFG and PA nano-graphite powders are composed of primary graphene flakes which are much smaller than that of the EG powder. The dispersibility of these nano-graphite powders was investigated in two different solvents (NMP, which is known as a good solvent and IPA, which is known as a poor solvent) by taking into account the centrifugation speed and the sonication power. The SEFG-IPA dispersion was divided into two parts; one part was centrifuged at $500 \mathrm{rpm}$ for $45 \mathrm{~min}$, while the other part was centrifuged at $900 \mathrm{rpm}$ for $45 \mathrm{~min}$ in order to observe the effect of centrifugation speed on the concentration of the graphene-based material remaining in the dispersion. UV-VIS absorption spectra of the dispersions prepared from SEFG and PA powders showed the characteristic peak of graphene at $\sim 266 \mathrm{~nm}$ and $\sim 281 \mathrm{~nm}$ for the IPA- and NMP-based dispersions, respectively, and the rest of the spectra were flat and featureless. The absorbance values at $660 \mathrm{~nm}$ were measured for the calculation of graphene-based material concentrations. Fig. 2(a) shows that SEFG-IPA and SEFGNMP dispersions resulted in higher concentration values than PA-IPA and PA-NMP dispersions, respectively. Especially, the concentration difference between SEFG-IPA and PA-IPA dispersions is significant. This showed that the nano-graphite powder derived from natural graphite provided higher concentrations with respect to nano-graphite powder derived from synthetic graphite, although SEFG has a much lower SSA than that of PA. Kozhemyakina et al. [17] reported that carbon uptake decreased with increasing SSA for the graphite materials with a SSA between 100 and $1200 \mathrm{~m}^{2} / \mathrm{g}$. The dispersibility of SEFG and PA powders in NMP is higher than the dispersibility of these powders in IPA, as expected, due to well matched surface energy of NMP to that of graphene and higher viscosity of NMP compared to that of IPA. SEFG-NMP120 min dispersion provided the maximum graphene-based material concentration $(\sim 1.22 \mathrm{mg} / \mathrm{ml})$ achieved in this study. However, SEFG-IPA-120 min and SEFG-IPA-90 min dispersions also resulted in relatively high concentrations $(\sim 1.16$ and $\sim 1.11 \mathrm{mg} / \mathrm{ml}$, respectively), although IPA is assumed as a poor solvent. These values are much higher than that of the concentration $(0.5 \mathrm{mg} / \mathrm{ml})$ reported by O'Neill et al. [15] who dispersed graphite powder in IPA for $48 \mathrm{~h}$ and subsequently centrifuged at $500 \mathrm{rpm}$. 

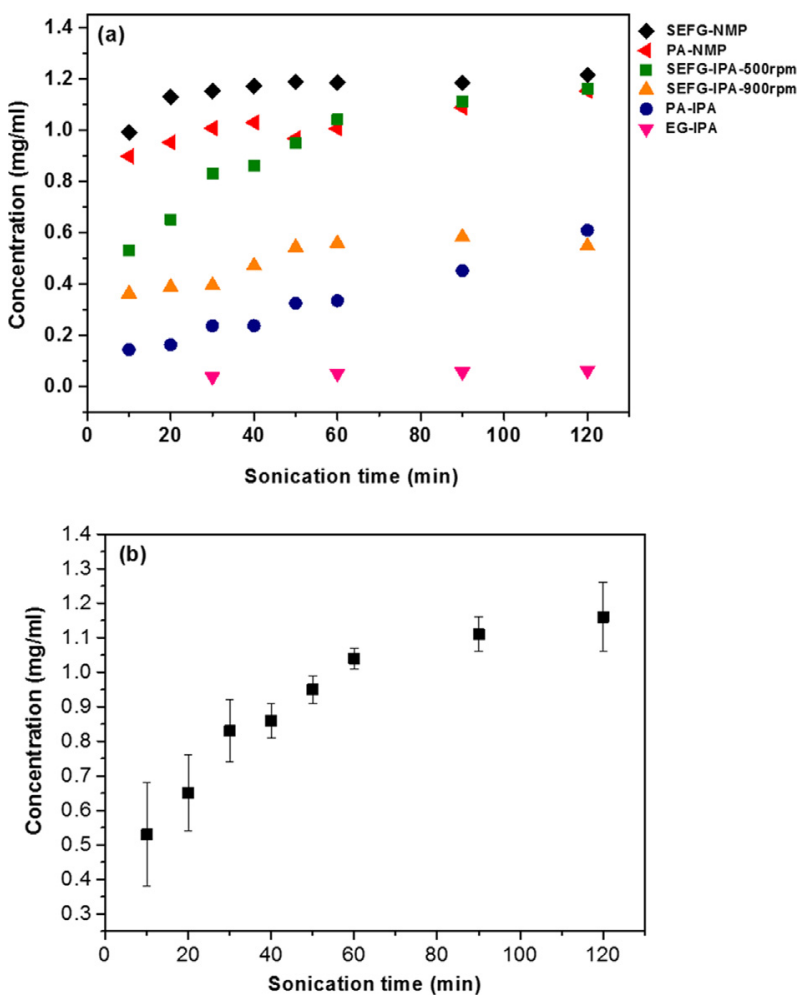

Fig. 2. Concentration of graphene-based material remaining after centrifugation at $500 \mathrm{rpm}$ for $45 \mathrm{~min}$ as a function of sonication time (a) for the IPA- and NMP-based dispersions prepared by bath sonication of SEFG and PA powders and a following centrifugation at $500 \mathrm{rpm}$ for $45 \mathrm{~min}$ (the results for the SEFG-IPA dispersions which were centrifuged at $900 \mathrm{rpm}$ for $45 \mathrm{~min}$ and the EG-IPA dispersions were also shown in the plot, for comparison), and (b) for the SEFG-IPA dispersions.

Fig. 2(b) shows the concentration vs. sonication time plot of the SEFG-IPA dispersions (centrifuged at $500 \mathrm{rpm}$ ) where the error bars represent the standard deviation calculated from at least three measurements. It should be noted here that the concentration values achieved at any sonication time show some variations depending on the equipment related variability, as in agreement with Khan et al. [12]. The authors reported that the sonic energy input to the sample is sensitive to the water level, the exact position in the bath, the volume of dispersion and vessel shape; moreover, sonic baths often have power outputs different to the rated power output. Consequently, this may cause variations in the concentration values attained at any sonication time, even if nominally identical baths are used [12]. In the present study, in order to minimize the variations arising from the ultrasonic bath, all the dispersions were prepared in the same volume (in $20 \mathrm{ml}$ vials by dispersing the powders in $10 \mathrm{ml}$ solvent), and the vials (4 vials at the same time) were placed at a certain position of the sonic bath up to a certain level of water in each time. The water level of the sonic bath was followed carefully since water evaporation occurs due to temperature increase in water during sonication, and the level of water was kept at a certain level.

Besides increasing graphene-based material concentration as much as possible, determination of the optimum sonication time is also critical in order to maintain graphene quality while increasing its concentration. Although long sonication times may provide a higher degree of exfoliation depending on the characteristics of the graphite-based precursor, it may also cause cutting of the graphene sheets into much smaller pieces and may introduce defects into them [23]. In this study, the optimum sonication time was determined as 90 min for further characterization and comparison of the dispersions that were prepared from different graphite- based precursors. The absorbance measurements and the concentration calculations revealed that high concentration graphenebased dispersions $(\sim 1.11 \mathrm{mg} / \mathrm{ml}$ ) can be prepared in a low boiling point solvent, IPA, in a short sonication time ( $\sim 90 \mathrm{~min})$ by using SEFG powder (SEFG-IPA-90 min sample) as starting material. It should also be noted that this concentration value was further increased up to $\sim 1.3 \mathrm{mg} / \mathrm{ml}$, when the sonication was performed in vials of $50 \mathrm{ml}$, instead of $20 \mathrm{ml}$.

Table 1 summarizes the concentration values of graphenebased materials in IPA- and NMP-based dispersions prepared from EG, SEFG or PA powders by sonication for $90 \mathrm{~min}$ and a following centrifugation. Although tip sonication was successfully applied to exfoliate EG and higher concentration values were obtained in comparison to that of bath sonication, it was not very efficient at exfoliating SEFG and PA powders. For the SEFG powder, the concentration of graphene-based material remaining in the dispersion after $90 \mathrm{~min}$ of sonication followed by centrifugation at $500 \mathrm{rpm}$ decreased from 1.11 to $0.83 \mathrm{mg} / \mathrm{ml}$, when tip sonication was used instead of bath sonication. For the PA powder, tip sonication and bath sonication provided similar concentration values. Concentration of the graphene-based material in the SEFG-IPA-90 min$900 \mathrm{rpm}$ dispersion was measured as $0.58 \mathrm{mg} / \mathrm{ml}$. The decrease in the concentration value with the increase in centrifugation speed was expected, since smaller and thinner flakes are kept in the dispersion at higher centrifugation speeds [12].

Fig. 3 shows FEG-SEM micrographs of the SEFG and PA powders before and after exfoliation in IPA for $90 \mathrm{~min}$ followed by centrifugation. The FEG-SEM micrograph of the as-received SEFG powder shows agglomerates with lateral dimensions of $\sim 1-4 \mu \mathrm{m}$ (Fig. 3 (a)). These agglomerates are mostly composed of graphene/graphite flakes which are $100-500 \mathrm{~nm}$ across, as well as a small amount of larger flakes up to a few $\mu \mathrm{m}$. Although sonication for 90 min in IPA provided dispersion of these agglomerates into smaller and thinner flakes to some extent, the agglomerated structure could not be prevented completely due to van der Waals forces between individual flakes (Fig. 3(b)). The FEG-SEM micrograph of the as-received PA powder shows a higher degree of agglomeration in comparison to the SEFG powder (Fig. 3(c)). This is due to higher SSA of the PA powder which is twice the SSA of the SEFG. Due to its smaller primary particle size, van der Waals forces between graphene layers are higher; therefore, the degree of agglomeration is expected to be higher than that of the SEFG. Sonication of the PA powder in IPA for 90 min successfully dispersed these agglomerates into smaller and thinner pieces (Fig. 3(d)).

\section{Size distribution and zeta potential measurements}

DLS analyses of the IPA-based dispersions of the SEFG and PA powders indicated a monomodal size distribution (Fig. 4). The dispersions prepared from the SEFG powder had broader size distributions and larger mean particle sizes than the dispersions

Table 1

Concentration and yield of graphene-based materials in IPA- and NMP-based dispersions prepared from EG, SEFG or PA powders by $90 \mathrm{~min}$ of sonication and a following centrifugation.

\begin{tabular}{lll}
\hline Sample code & Concentration $(\mathrm{mg} / \mathrm{ml})$ & Yield $(w t \%)$ \\
\hline EG-IPA-90 min & 0.04 & 20 \\
EG-IPA-90 min-TS & 0.06 & 30 \\
SEFG-IPA-90 min & 1.11 & 37 \\
SEFG-IPA-90 min-TS & 0.83 & 28 \\
SEFG-IPA-90 min-900 rpm & 0.58 & 19 \\
SEFG-NMP-90 min & 1.18 & 39 \\
PA-IPA-90 min & 0.45 & 15 \\
PA-IPA-90 min-TS & 0.52 & 17 \\
PA-NMP-90 min & 1.09 & 36 \\
\hline
\end{tabular}



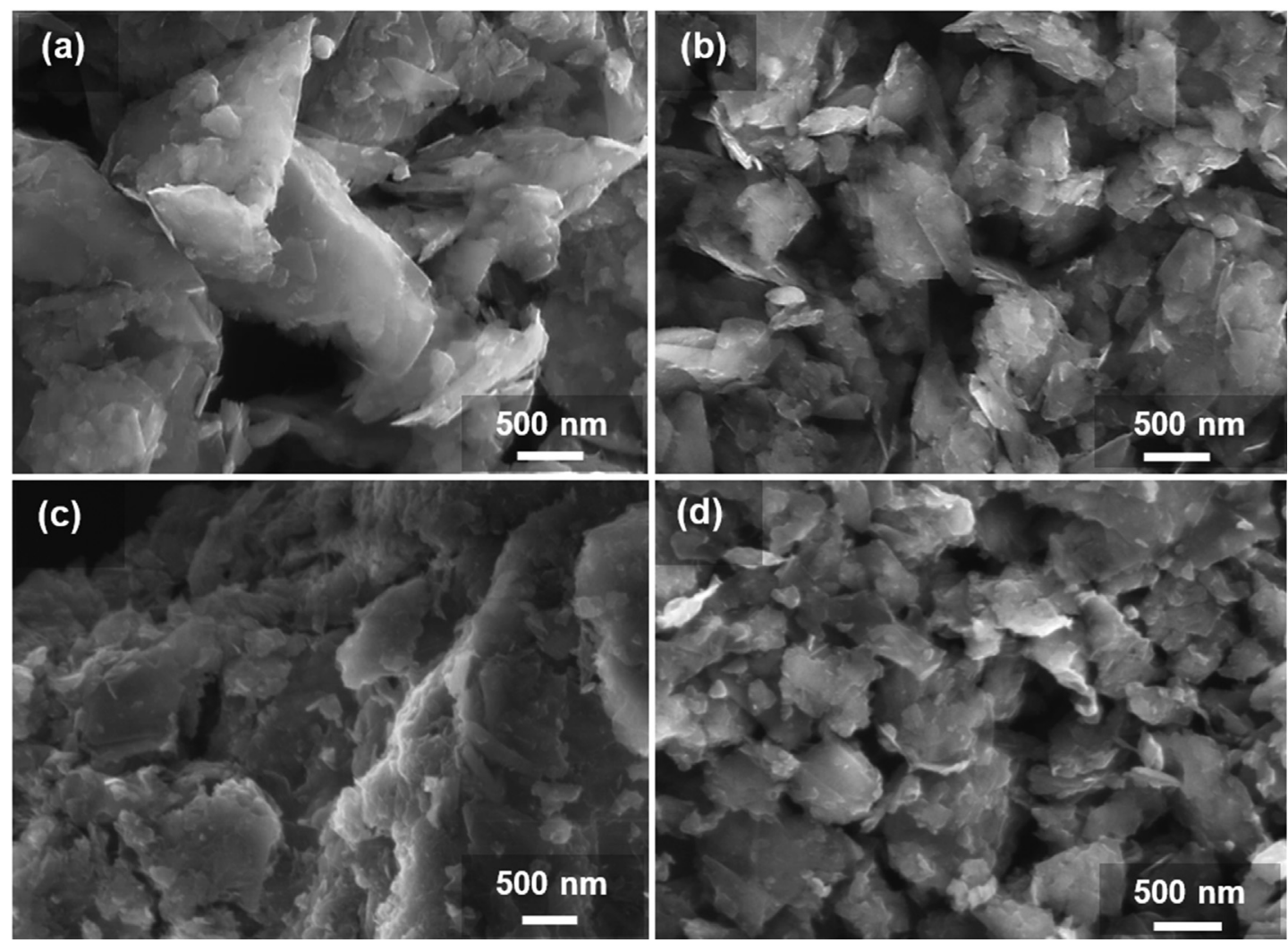

Fig. 3. FEG-SEM micrographs of (a) SEFG, (b) SEFG-IPA-90 min, (c) PA, and (d) PA-IPA-90 min.
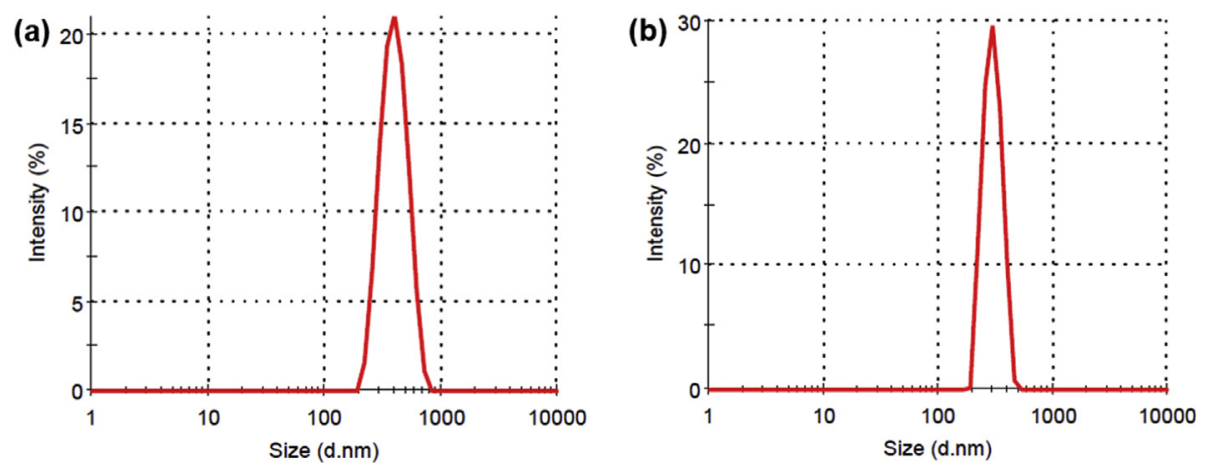

Fig. 4. Particle size distributions of the (a) SEFG-IPA-90 min and (b) PA-IPA-90 min.

prepared from the PA powder (Fig. 4(a) and (b)). The lateral size (hydrodynamic diameter) of the graphene/graphite flakes in the SEFG-IPA-90 min dispersion ranged between 200 and $800 \mathrm{~nm}$ with an average size of $403 \mathrm{~nm}$ (Fig. 4(a)). The PA-IPA-90 min dispersion resulted in graphene/graphite flakes with a narrower size distribution (between 200 and $500 \mathrm{~nm}$ ) and a smaller average lateral size of $299 \mathrm{~nm}$ (Fig. 4(b)). The DLS measurements of the dispersions prepared from the EG powder could not be performed, since their polydispersity index was very high. This means that these dispersions had a very broad size distribution and may contain large particles or agglomerates; therefore, may not be appropriate for DLS measurements. The sedimentation measurements also showed that the dispersions prepared from the EG powder precipitated completely within 2 days, indicating a very poor stability, in good agreement with the DLS particle size analysis results.

Zeta potential measurements were performed at the natural $\mathrm{pH}$ of the dispersions at $25^{\circ} \mathrm{C}$. It should be noted that these measurements were done a few months later than the dispersions were prepared. They were diluted and mildly sonicated for $\sim 30 \mathrm{~s}$ just before the measurements. IPA-based dispersions of SEFG and PA showed zeta potential values between $-30 \mathrm{mV}$ and $-40 \mathrm{mV}$ indicating moderate stability according to ASTM [24].

The sedimentation measurements revealed that the dispersions prepared from the PA powder show relatively high stability due to narrower size distribution and smaller mean particle size of the PA starting powder in comparison to the SEFG. The SEFG-IPA-90 min sample, which exhibited the highest concentration among the prepared IPA-based dispersions, revealed a moderate stability with $35 \%$ non-sedimenting few-layer graphene sheets remaining in the dispersion after 8 weeks, in agreement with zeta potential measurements. However, the non-sedimenting graphene concentration $(0.39 \mathrm{mg} / \mathrm{ml})$ of this sample was still higher than that of the other dispersions prepared in IPA, when the initial concentration of the dispersions was taken into account (see Supplementary Information, Fig. S1 and Table S1). 
Raman analyses of the starting powders and the exfoliated materials

Raman spectroscopy, which provides a fast, high-throughput and nondestructive identification of graphene layers [25] was used to evaluate the number of layers and the quality of the prepared graphene-based materials. Raman analyses were performed on the starting materials and their dispersions in IPA. Fig. 5 shows the representative Raman spectra of the expandable graphite, expanded graphite (EG) and the exfoliated material (EG-IPA90 min-TS). Table 2 gives a summary of the corresponding Raman characteristics. Raman spectra of all the samples shown in Fig. 5 include a strong G-band at $1582 \mathrm{~cm}^{-1}$, which arises from the inplane $\mathrm{C}-\mathrm{C}$ bond stretching in graphitic materials and is common to all $\mathrm{sp}^{2}$-bonded carbon systems [26], and a second order G'band, also named 2D-band, at $\sim 2720 \mathrm{~cm}^{-1}$. The spectra of the expandable graphite and the EG-IPA-90 m-TS samples also include disorder induced D-band at $\sim 1350 \mathrm{~cm}^{-1}$ and $\mathrm{D}^{\prime}$-band at $\sim 1620 \mathrm{~cm}^{-1}$.

The 2D-band is a second-order process related to a phonon near the $\mathrm{K}$ point in graphene, activated by double resonance process, which is responsible for its dispersive nature and cause a strong dependence on any perturbation on the electronic and/or phonon structure of graphene [26]. Therefore, the 2D-band changes in line-shape, line-width and peak position when the number of graphene layers increases in a Bernal stacked graphene [27]. It should be also noted that this two-phonon band is allowed in the second order Raman spectra of graphene without any kind of disorder or defects [28]. Raman measurements which were performed on different regions of expandable graphite revealed variations in the line-shape of the 2D-band. Fig. 6 and Table 3 show the curve fittings and the fitting parameters of the 2D-bands of expandable graphite, EG and EG-IPA-90 min-TS samples, respectively. Although the 2D-band of expandable graphite could be generally fit using two Lorentzian peaks revealing a graphite structure (Fig. 6(a), Table 3), some spectra needed three Lorentzian peaks to fit the 2D-band of expandable graphite (Fig. 6(b)). The latter case was accompanied by a relatively higher intensity D-band at $\sim 1350 \mathrm{~cm}^{-1}$ and the appearance of the $\mathrm{D}^{\prime}$-band at $\sim 1620 \mathrm{~cm}^{-1}$ compared to the former (Fig. 5(a) and (b)), and supposed to be recorded from flake edges rather than basal plane of the expandable graphite. D-band arises from breathing modes of $\mathrm{sp}^{2}$ atoms in rings and requires a defect for its activation $[11,26]$. Hence, it

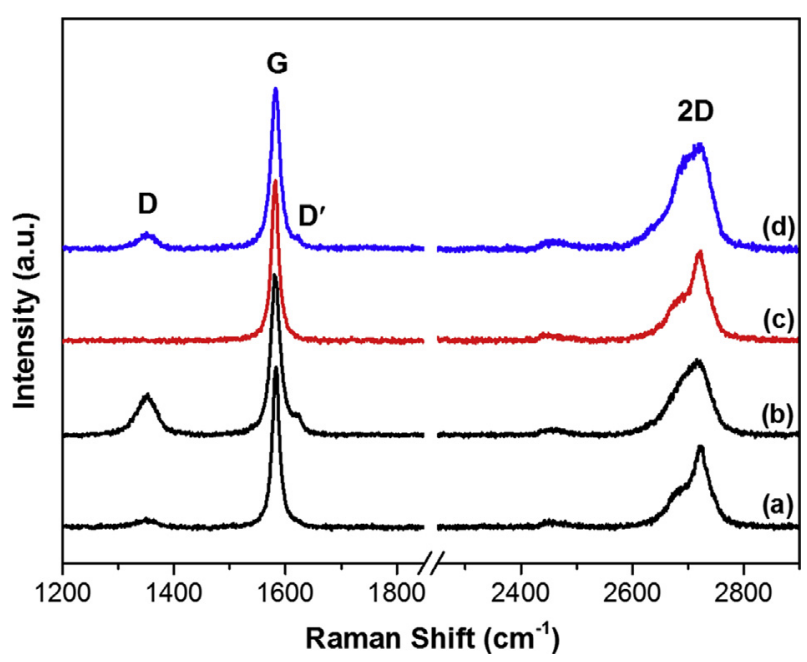

Fig. 5. Representative Raman spectra (normalized to the G-band) of (a), (b) expandable graphite ( $(a)$ and (b) show the Raman spectra recorded at different locations of the laser spot over the thin film), (c) expanded graphite (EG), and of (d) exfoliated (EG-IPA-90 min-TS) sample. is observed in case of a disordered sample and/or at the edge of the sample [28]. The presence of disorder/defect induced D- and $\mathrm{D}^{\prime}$-bands in the Raman spectrum of expandable graphite can be generally attributed to lattice deformation since the acid intercalation causes symmetry changes at a graphitic layer adjacent to an intercalant layer [29,30], as well as to edge distortion.

The 2D-band of the EG exhibited two-peaks profile, typical to graphite (Fig. 6(c)). The line-shape of the 2D-bands recorded from different locations of the EG-IPA-90 min-TS sample showed variations, indicating that the exfoliated sample is composed of few- or multi-layer graphene sheets, as well as graphite flakes. This result is consistent with the DLS particle size analysis and the sedimentation results, which revealed a polydisperse character for this sample. This may indicate that some of the expanded graphite remained in the dispersion without exfoliation or reagglomerated during sample preparation. The line-shape of the 2D-band shown for EG-IPA-90 min-TS in Fig. 6(d) is asymmetric; however, different from that of graphite. This peak was best fit with eight Lorentzian components with a fixed FWHM of $24 \mathrm{~cm}^{-1}$ (Table 3) and could be attributed to few-layer (3-5 layers) graphene. The $\pi$ electronic structure of graphene splits when a second layer is added. With an increase in the number of layers, the number of double resonance scattering processes increases, and eventually the line-shape converges to graphite, where only two peaks are observed [26]. Raman spectrum becomes hardly distinguishable from that of bulk graphite for more than five layers [25]. Malard et al. [28] reported that to correctly identify the line-shape of 2D-band for trilayer graphene, one should model the double resonance process by considering 15 different transitions. However, the energy separations of many of these fifteen different processes can be very close to each other from an experimental point of view. The authors reported that minimum number of peaks with a FWHM of $\sim 24 \mathrm{~cm}^{-1}$ is necessary to correctly fit the 2D-band, and splitting of 2D-band of trilayer graphene needs at least six peaks with a FWHM of $\sim 24 \mathrm{~cm}^{-1}$ for laser energies $\leqslant 2.41 \mathrm{eV}$ [28].

The intensity ratio of the D-band to that of the G-band $\left(I_{\mathrm{D}} / I_{\mathrm{G}}\right)$ is generally used to characterize defect content [26]. Fig. 7 shows the statistical histograms of the $I_{\mathrm{D}} / I_{\mathrm{G}}$ ratio for the expandable graphite, EG powder and EG-IPA-90 min-TS sample. The $I_{\mathrm{D}} / I_{\mathrm{G}}$ ratio of the expandable graphite ranged from $\sim 0.01$ to $\sim 0.45$ with a mean value of $\sim 0.13$ (Fig. 7(a)). When expandable graphite was subjected to a thermal expansion, the D-band almost disappeared and an extremely low $I_{\mathrm{D}} / I_{\mathrm{G}}$ ratio ( $\sim 0.01$ in average) was found for EG, indicating removal of the defects arising from acid intercalation (lattice deformation) (Fig. 7(b)). The $I_{\mathrm{D}} / I_{\mathrm{G}}$ ratio of the EG-IPA90 min-TS ( $\sim 0.06$ in average) showed a slight increase in comparison to that of the EG powder, indicating that exfoliation process introduced some defects to the graphene/graphite flakes (Fig. 7 (c)). These defects may be either point defects in the basal planes or may arise from the flake edges $[12,15,23]$. For solvent exfoliated graphene flakes, the defects are generally attributed to the new edges which are created as a result of cutting initially large crystallites into smaller flakes by sonication and act as defects $[11,23,31]$. However, this $I_{\mathrm{D}} / I_{\mathrm{G}}$ ratio is still significantly low, revealing high quality of the exfoliated flakes.

Fig. 8(a) shows the Raman spectra of the thin films prepared from the SEFG starting powder and its IPA-based dispersion (SEFG-IPA-90 m). The spectrum of the SEFG-IPA-90 $\mathrm{m}$ sample annealed at $2600{ }^{\circ} \mathrm{C}$ in $\mathrm{Ar}$ atmosphere is also presented in Fig. 8 (a). The corresponding Raman characteristics are given in Table 2. A red-shift was observed in all the band-positions of these samples compared to that of the EG-based samples, accompanied by a broadening of the FWHM (Table 2). The 2D-band line-shapes of all the samples, including the SEFG starting powder, are distinctly different from that of graphite and were best fit with eight Lorent- 
Table 2

Raman features of the starting powders (EG, SEFG and PA) and of the thin films prepared from IPA-based dispersions of these powders. ${ }^{\dagger}$

\begin{tabular}{|c|c|c|c|c|c|c|c|c|}
\hline & \multirow{2}{*}{$\begin{array}{l}\text { D-band } \\
\text { Position }(\omega) \\
\left(\mathrm{cm}^{-1}\right)\end{array}$} & \multicolumn{2}{|l|}{ G-band } & \multicolumn{2}{|c|}{ 2D-band } & \multirow[t]{2}{*}{$\mathrm{I}_{\mathrm{D}} / \mathrm{I}_{\mathrm{G}}$} & \multirow[t]{2}{*}{$\mathrm{I}_{2 \mathrm{D} /} / \mathrm{I}_{\mathrm{G}}$} & \multirow[t]{2}{*}{ Estimated number of layers } \\
\hline & & $\begin{array}{l}\text { FWHM } \\
\left(\mathrm{cm}^{-1}\right)\end{array}$ & $\begin{array}{l}\text { Position }(\omega) \\
\left(\mathrm{cm}^{-1}\right)\end{array}$ & $\begin{array}{l}\text { FWHM } \\
\left(\mathrm{cm}^{-1}\right)\end{array}$ & $\begin{array}{l}\text { Position }(\omega) \\
\left(\mathrm{cm}^{-1}\right)\end{array}$ & & & \\
\hline Expandable graphite (a) & 1351 & 17 & 1582 & 53 & - & 0.08 & - & - \\
\hline Expandable graphite (b) & 1353 & 21 & 1582 & 71 & 2717 & 0.19 & 0.46 & - \\
\hline $\mathrm{EG}$ & 1350 & 17 & 1582 & 56 & - & 0.01 & - & - \\
\hline EG-IPA-90 min-TS & 1353 & 18 & 1582 & 68 & 2720 & 0.07 & 0.60 & Few-layer (3-5 layers) \\
\hline $\mathrm{SEFG}^{*}$ & 1345 & 24 & 1574 & 77 & 2696 & 0.26 & 0.37 & Agglomerates of few-layers \\
\hline SEFG-IPA-90 min & 1346 & 23 & 1573 & 75 & 2697 & 0.24 & 0.38 & Few-layer (3-5 layers) \\
\hline SEFG-IPA-90 min- $2600{ }^{\circ} \mathrm{C}$ & 1344 & 23 & 1572 & 75 & 2692 & 0.07 & 0.43 & Few-layer (3-5 layers) \\
\hline $\mathrm{PA}^{* *}$ & 1344 & 30 & 1575 & 78 & 2692 & 0.39 & 0.43 & Agglomerates of few-layers \\
\hline PA-IPA-90 min & 1343 & 28 & 1571 & 76 & 2685 & 0.30 & 0.43 & Few-layer (3-5 layers) \\
\hline
\end{tabular}

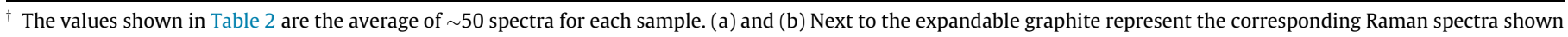
in Fig. 5

* Surface enhanced flake graphite.

*** Primary artificial.
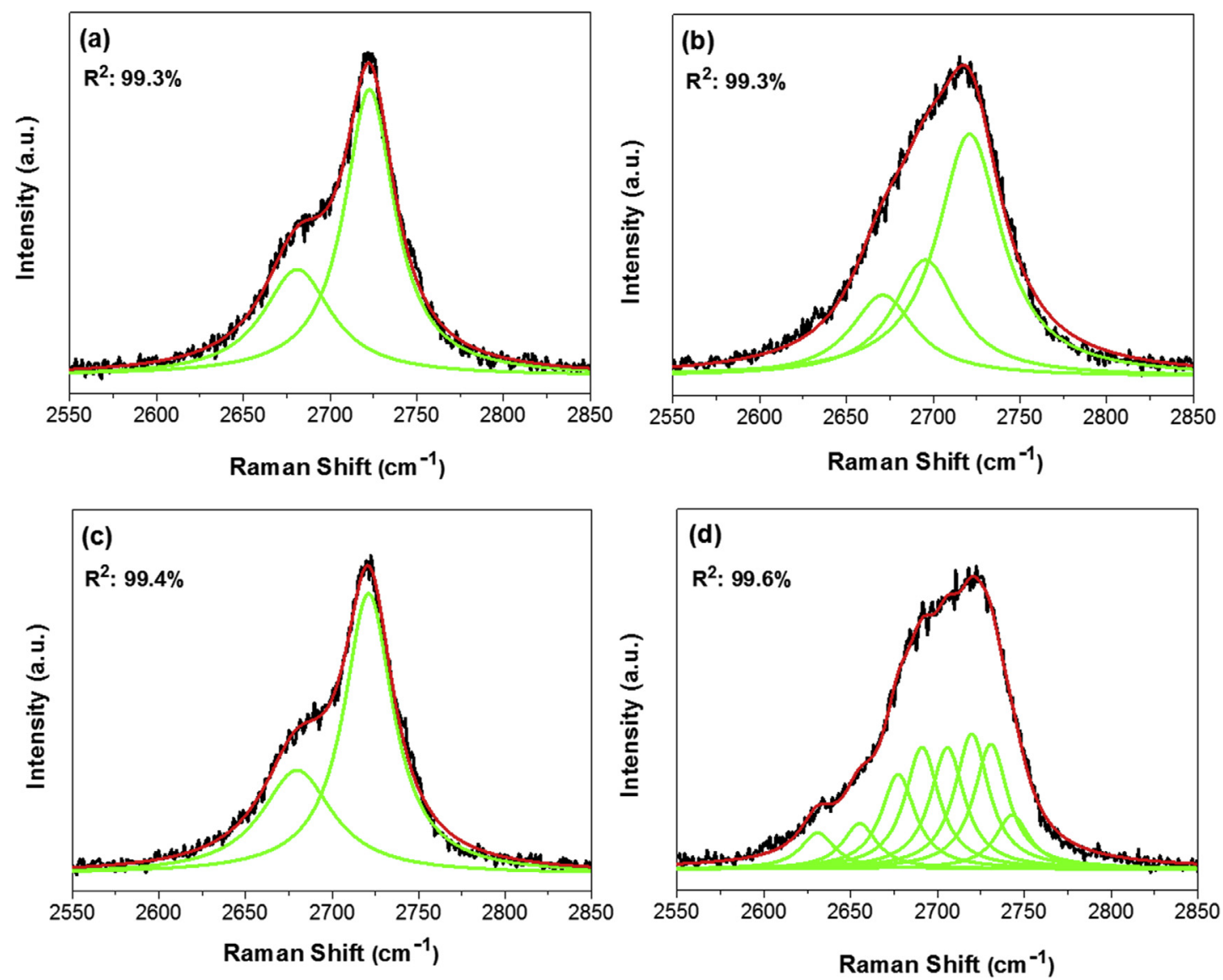

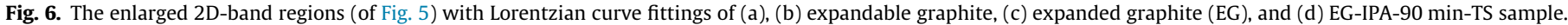
The black, green and the red curves represent the spectra, fit peaks and the cumulative fit peak, respectively.

Table 3

Fitting components of the 2D-bands of the corresponding spectra shown in Fig. 5. The value in parentheses is the FWHM ( $\mathrm{cm}^{-1}$ ) of the corresponding fit peak.

\begin{tabular}{|c|c|c|c|c|c|c|c|c|}
\hline & Peak $1\left(\mathrm{~cm}^{-1}\right)$ & Peak $2\left(\mathrm{~cm}^{-1}\right)$ & Peak $3\left(\mathrm{~cm}^{-1}\right)$ & Peak $4\left(\mathrm{~cm}^{-1}\right)$ & Peak $5\left(\mathrm{~cm}^{-1}\right)$ & Peak $6\left(\mathrm{~cm}^{-1}\right)$ & Peak $7\left(\mathrm{~cm}^{-1}\right)$ & Peak $8\left(\mathrm{~cm}^{-1}\right)$ \\
\hline Expandable graphite (a) & - & - & $2681(35)$ & - & - & $2723(48)$ & - & - \\
\hline Expandable graphite (b) & - & - & $2671(45)$ & $2696(45)$ & - & $2721(45)$ & - & - \\
\hline EG & - & - & $2680(35)$ & - & - & $2721(50)$ & - & - \\
\hline EG-IPA-90 min-TS & $2631(24)$ & $2655(24)$ & $2677(24)$ & $2691(24)$ & $2706(24)$ & $2720(24)$ & $2731(24)$ & $2743(24)$ \\
\hline
\end{tabular}



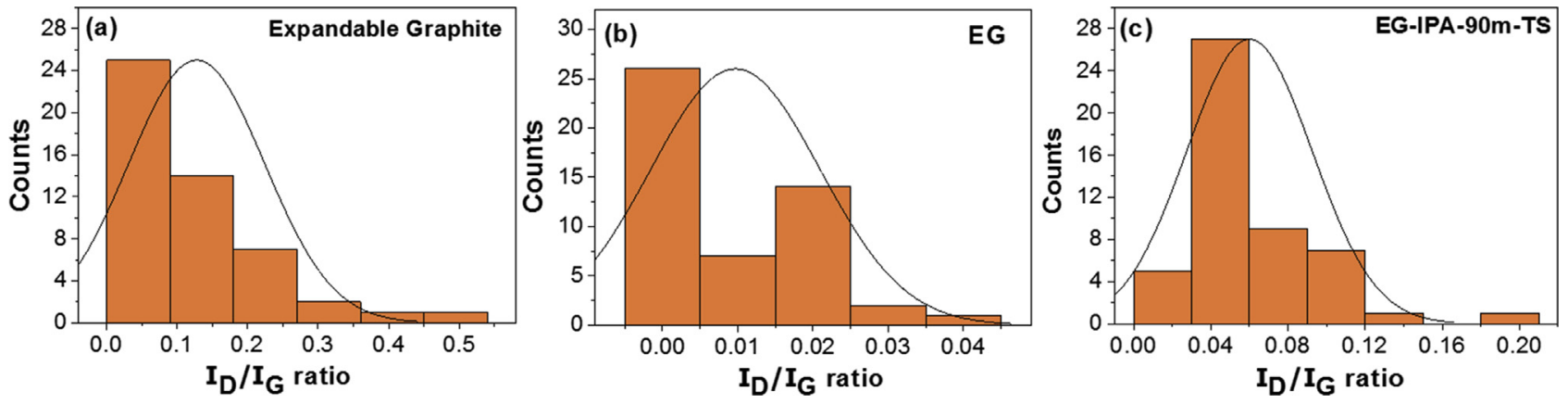

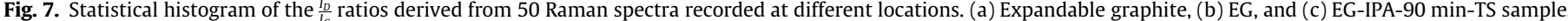
The distribution curves indicate the mean of the data.
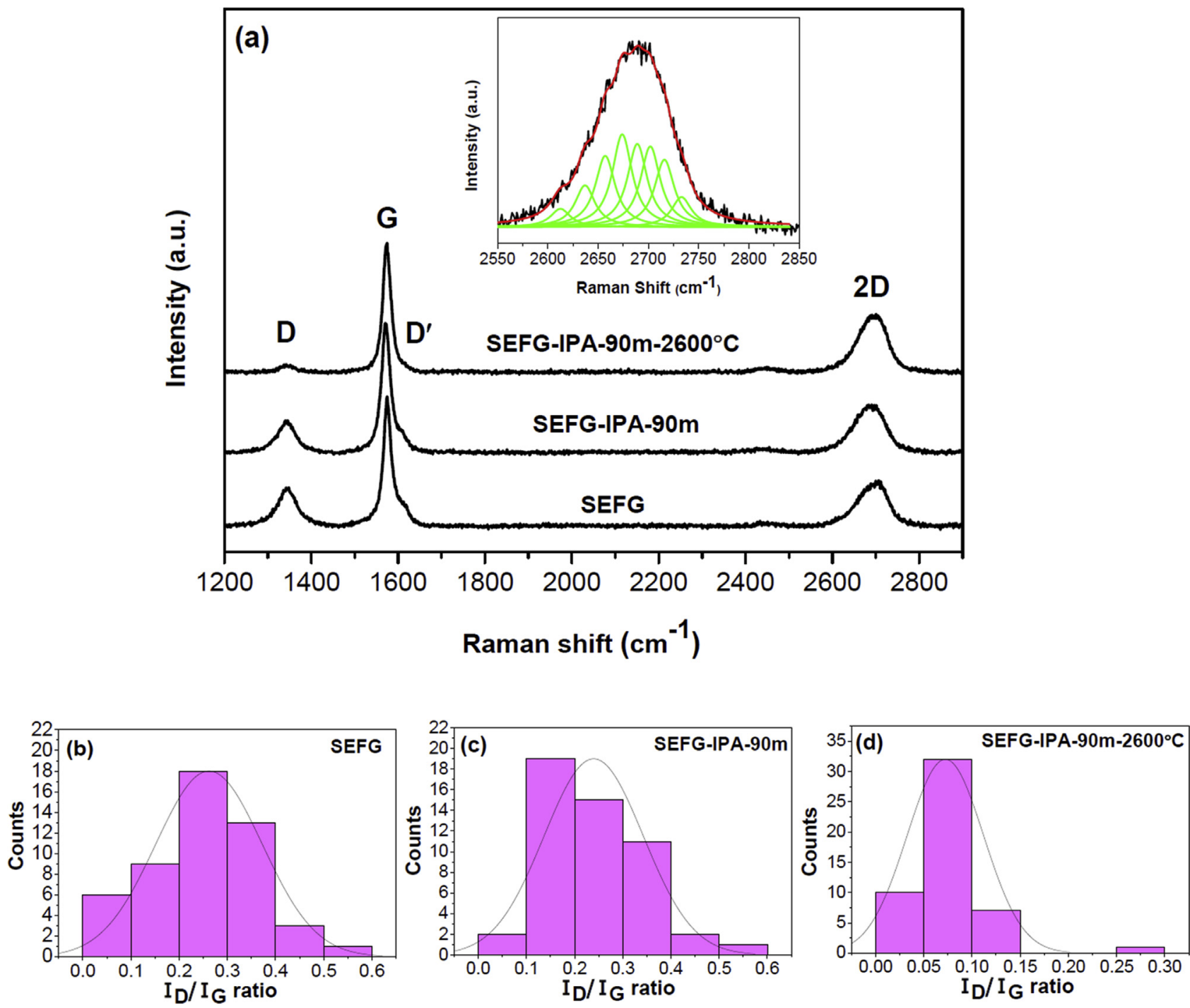

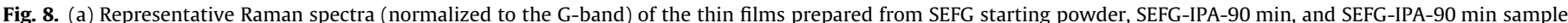

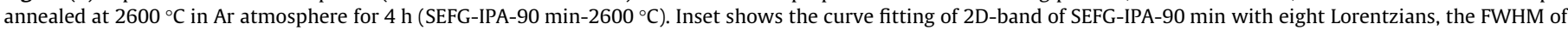

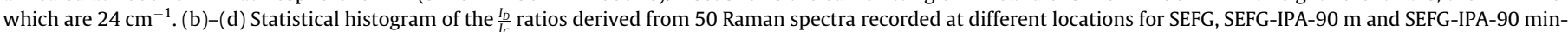
$2600{ }^{\circ} \mathrm{C}$ samples, respectively. The distribution curves indicate the mean of the data.

zian peaks, each with a FWHM of $\sim 24 \mathrm{~cm}^{-1}$ (similar to the case observed for the EG-IPA-90 min sample as discussed previously), indicating that the number of layers of individual flakes are mostly $3-5$. The representative fitting curve of the 2D-band of the SEFGIPA-90 min sample is shown in the inset of Fig. 8. The correspond- ing fitting parameters are presented in Table S2. These fitting results were interpreted as the SEFG starting material, which is a nano-graphite powder produced by subjecting natural graphite to many processing steps in order to increase its SSA to $\sim 175 \mathrm{~m}^{2} / \mathrm{g}$, is composed of agglomerates of individual few-layer 
graphene sheets mostly, while the exfoliation process helps to disperse these agglomerates.

Fig. 8(b)-(d) show statistical histograms of the $I_{\mathrm{D}} / I_{\mathrm{G}}$ ratio for the SEFG starting powder, SEFG-IPA-90 min and SEFG-IPA-90 min$2600{ }^{\circ} \mathrm{C}$ samples, respectively. The $I_{\mathrm{D}} / I_{\mathrm{G}}$ ratio of the SEFG starting powder varied between 0.05 and 0.53 with a mean value of 0.26 , while $I_{\mathrm{D}} / I_{\mathrm{G}}$ ratio of the SEFG-IPA-90 min sample varied between 0.08 and 0.60 with a mean value of 0.24 , which is comparable to those reported in the literature for liquid phase exfoliated graphite $(<0.5)[12,15,32]$. The similar $I_{\mathrm{D}} / I_{\mathrm{G}}$ ratio observed for the starting powder and the exfoliated samples may indicate that the defects in the exfoliated samples originate from the starting powder, rather than introduction of new defects (such as flake edges) into the sample during exfoliation process. Annealing of the SEFGIPA-90 min sample at $2600{ }^{\circ} \mathrm{C}$ in $\mathrm{Ar}$ atmosphere for $4 \mathrm{~h}$ resulted in a significant decrease in the $I_{\mathrm{D}} / I_{\mathrm{G}}$ ratio, which ranged between 0 and 0.26 with a mean value of 0.07 , as shown by the corresponding statistical histogram (Fig. 8(d)), and also in disappearance of the $\mathrm{D}^{\prime}$-band (corresponding Raman spectrum in Fig. 8(a)). These were attributed to elimination of most of the defects/disorders in the SEFG-IPA-90 min sample and recovery of it as a result of annealing, confirming that the defects are mainly structural imperfections which most probably originate from the starting powder. These defects/disorders could be introduced into the SEFG powder during its production process as the point defects in the basal planes and/or the chemical residues.

Fig. 9(a) shows the Raman spectra of the thin films of the PA starting powder and the PA-IPA-90 min dispersion. Raman features of these samples are summarized in Table 2. 2D-bands of both PA and PA-IPA-90 min were best fit with eight Lorentzians with a FWHM of $24 \mathrm{~cm}^{-1}$, similar to those observed for the SEFG and its related dispersions, indicating the presence of few-layer (3-5 layers) graphene flakes in these samples. Inset in Fig. 9(a) shows the 2D-band fitting of the PA-IPA-90 min sample, and Table S2 gives the corresponding fitting components. Fig. 9(b) and (c) shows the statistical histograms of the $I_{\mathrm{D}} / I_{\mathrm{G}}$ ratio for the PA starting powder and the PA-IPA-90 min sample, respectively. The $I_{\mathrm{D}} / I_{\mathrm{G}}$ ratio of the PA powder ranged between 0.15 and 1.0 with a mean value of 0.39 , while the $I_{\mathrm{D}} / I_{\mathrm{G}}$ ratio of the PA-IPA- $90 \mathrm{~min}$ was in the range of $0.12-0.58$ with a mean value of 0.30 . These defect contents are higher than those observed for the SEFG powder and its dispersions. PA-IPA-90 $\mathrm{m}$ sample exhibited a narrower $I_{\mathrm{D}} / I_{\mathrm{G}}$ ratio distribution and a lower average $I_{\mathrm{D}} / I_{\mathrm{G}}$ ratio compared to the PA powder, indicating that the defect content of the starting powder is higher than that of the exfoliated sample. Both basal plane defects and/or flake edges could be responsible for this defect content. The PA is a nano-graphite powder with a very high SSA $\left(350 \mathrm{~m}^{2} / \mathrm{g}\right)$, about twice that of the SEFG powder, resulting in a higher degree of agglomeration of the graphene/graphite flakes compared to the SEFG powder. Due to this agglomeration, more flake edges could have encountered with the laser spot during Raman analysis, giving rise to D and $\mathrm{D}^{\prime}$-bands. In addition, the imperfections such as the point defects in the basal planes and/ or the chemical residues could have been introduced into the PA powder during its production from synthetic graphite. The reason of decreasing defect amount with exfoliation could be the dispersion of the agglomerates, therefore, decreasing the flake concentration in the area of the laser spot and/or removal of some of the defected flakes or impurities, present in the starting powder, by centrifugation.

\section{TEM analyses of the starting powders and the exfoliated materials}

In order to determine the number of graphene layers and their orientation with respect to each other, TEM and high resolution TEM (HRTEM) analyses were performed. Fig. 10(a) shows a repre- sentative low magnification TEM image of the graphene flakes in the EG-IPA-90 min-TS sample. The lateral sizes of the flakes are relatively large, mostly a few micrometers. Folded and wrinkled regions are observed in most of the flakes (dashed arrows point out the folded regions, solid arrows point out the wrinkles). The color contrast in the region shown by the dashed rectangle in Fig. 10(a) reveals single- or bi-layer graphene at the bottom and many other layers which are piled disorderedly on top of it. Fig. 10(b) shows HRTEM image of the EG-IPA-90 min-TS sample, revealing highly crystalline structure of the graphene-based material obtained by exfoliation of expanded graphite. Corresponding fast Fourier transform (FFT) (at the insets in Fig. 10(b)) shows hexagonal spot patterns indicating the sixfold symmetry feature of graphene. Diffraction patterns and FFTs are also utilized to identify and observe misoriented (turbostratic) graphene sheets [33]. HRTEM analyses of the EG-IPA-90 min-TS sample revealed turbostratic graphene (Fig. 10(c)), as well as ordered (Bernal stacked) graphene sheets as shown in Fig. 10(b). Turbostratic graphene reveals itself in the diffraction pattern or in the FFT by multiple diffraction spots [34]. Rather than having only 6 spots in each ring of the pattern as in the case for single and AB-stacked few layer graphene, several orders of 6 spots $(6,12,18$ etc.) appear for misoriented layers [33]. The FFT (at the inset) of the corresponding HRTEM image shown in Fig. 10(c) indicates a turbostratic graphene sheet, the rotation angle of which is $\sim 10.5^{\circ}$. The misalignment in between layers might be a result of restacking of layers with different orientations on top of each other during exfoliation or folding of layers [33]. It should be also noted that the HRTEM image in Fig. 10(c) indicates that the flake surface is not uniform and an amorphous layer is present on the top. This could be due to residual solvent (IPA). HRTEM analysis also revealed the presence of graphite flakes ( $>10$ layers) (not shown), as well as few layer $(<5$ layers) and turbostratic graphene sheets.

Low magnification TEM micrographs of the starting SEFG powder showed agglomerates/aggregates of graphene/graphite flakes with some impurities on their surface and vicinity. Exfoliation of this powder dispersed these agglomerates to some extent and provided mostly few-layer graphene flakes with a lateral size of a few hundred nanometers. These flakes are mostly folded, scrolled and entangled each other (Fig. 11(a)). Wang et al. [35] reported that corrugation and scrolling are part of the intrinsic nature of graphene nanosheets, which result from the fact that the $2 \mathrm{D}$ membrane structure becomes thermodynamically stable via bending. Fig. 11(b) and (c) show representative HRTEM micrographs of the flakes deposited from the SEFG-IPA-90 $\mathrm{m}$ dispersion. FFT images of these micrographs are shown in the insets. HRTEM micrographs revealed mostly few-layer ( $<5$ layers) graphene flakes, in agreement with Raman analyses, although sheets with more than 10 layers were also rarely observed. Fig. 11(b) shows a bilayer graphene, the edge of which was folded back allowing for a crosssectional view. The FFT of this image shows one set of hexagonal spots confirming the six-fold symmetry of graphene and suggesting that the fold back part has the same orientation as the original one [36]. Fig. 11(c) shows several few-layer graphene flakes as well as large, bended and folded graphite flakes which are randomly oriented with respect to each other, as confirmed by the multiple diffraction spots in the FFT (inset in Fig. 11(c)). HRTEM analyses also revealed an amorphous layer covering the surface of some flakes. This can be attributed to residual solvent (IPA) or to impurities present in the starting powder, in agreement with the Raman analyses which revealed a relatively high amount of defects for the SEFG powder. HRTEM micrograph and its corresponding FFT image shown in Fig. 11(d) revealed that the SEFG-IPA-90 min sample preserves its highly crystalline structure annealing at $2600{ }^{\circ} \mathrm{C}$ in $\mathrm{Ar}$ atmosphere for $4 \mathrm{~h}$. This confirms the Raman analysis results which indicated that the defects/disorders and impurities in the 

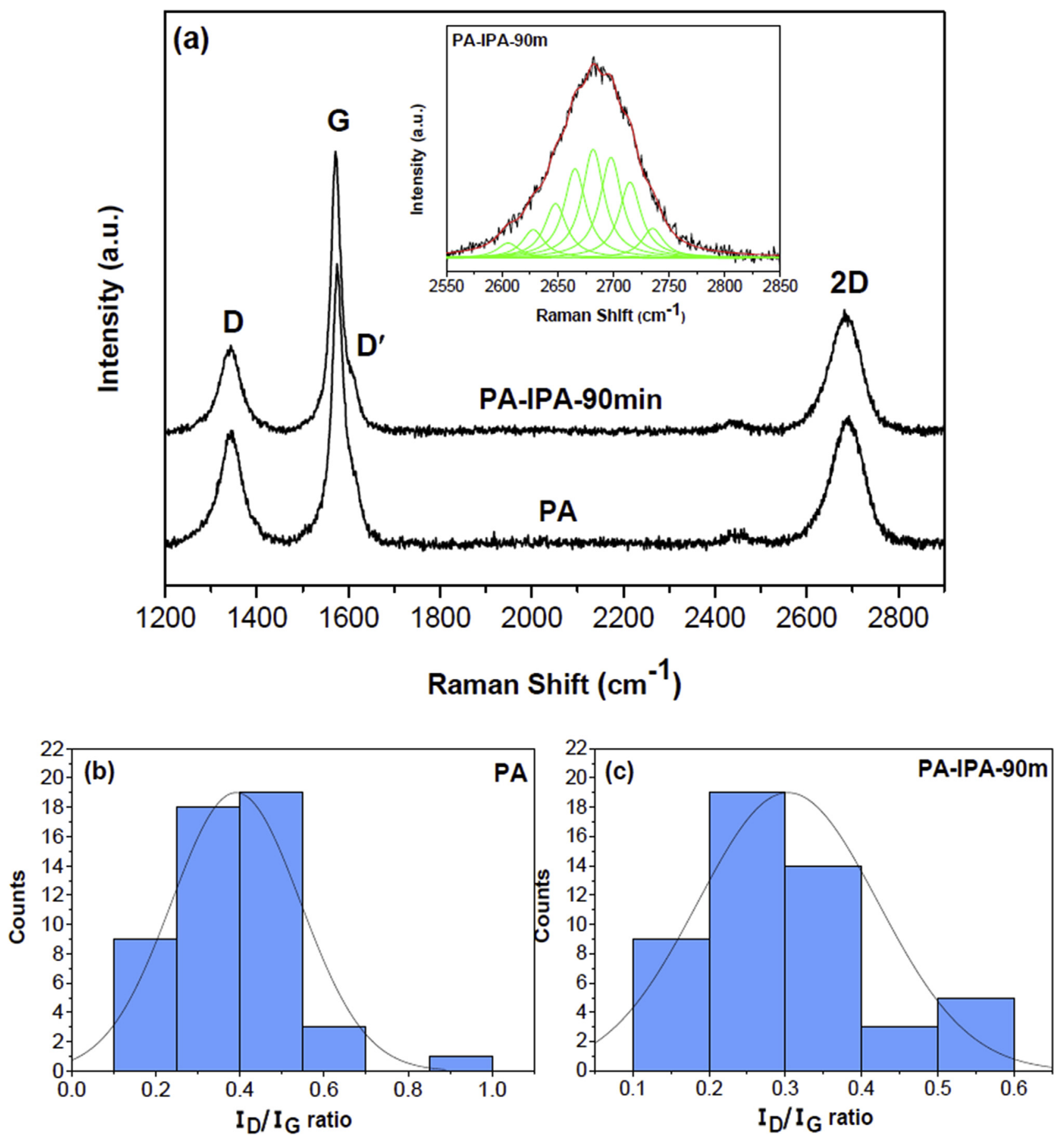

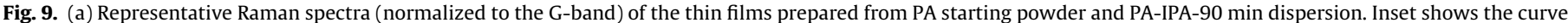

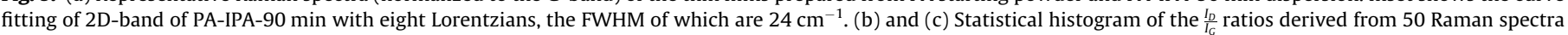
recorded at different locations for PA and PA-IPA-90 min, respectively. The distribution curves indicate the mean of the data.

exfoliated sample mainly arise from the SEFG starting powder and are eliminated and/or recovered by annealing.

Low magnification TEM micrograph of the PA nano-graphite powder revealed agglomerates/aggregates of graphite/graphene flakes. The exfoliation process dispersed these agglomerates to some extent similar to the exfoliation of SEFG and TEM evidenced graphene-like sheets with a smaller lateral size in comparison to those derived from SEFG (Fig. 12(a)), in agreement with the DLS analyses. However, large agglomerates were still observed after exfoliation of the PA powder due to relatively high SSA $\left(350 \mathrm{~m}^{2} /\right.$ g) of the PA powder that leads to higher van der Waals forces.
HRTEM micrographs revealed the edge details of the exfoliated flakes indicating mostly few-layer graphene sheets ( $<5$ layers), confirming the Raman analyses, as well as few amount of graphite flakes with more than 10 layers (Fig. 12(b)). As shown by the low magnification TEM images, the exfoliated graphene sheets are reaglomerated and entangled each other. Impact of the exfoliation process can be realized by observation of a flake edge along which the number of layers show variation, as indicated by a dashed circle in Fig. 12(b). A non-uniformity over some flake surfaces was also observed during HRTEM analysis (Fig. 12(b)). The respective FFT pattern of the region enclosed by a dashed square exhibited 


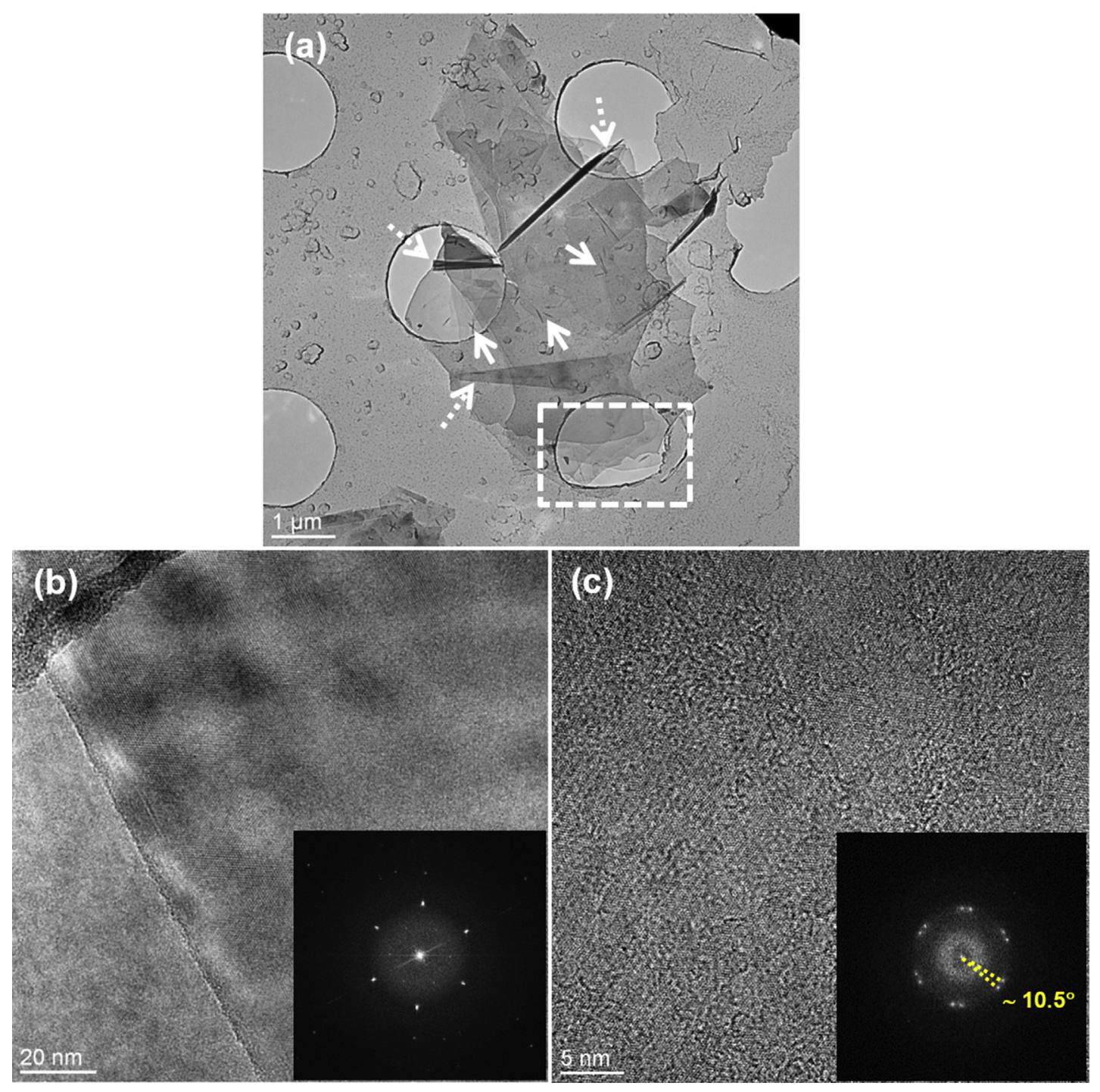

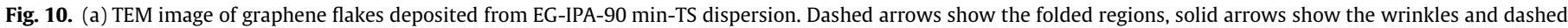

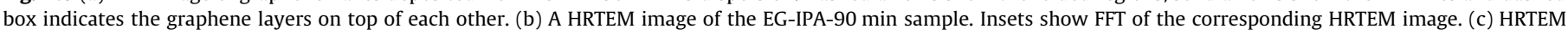
image of a turbostratic graphene showing an amorphous layer covering the surface. It's FFT (inset) indicates a rotation angle of $10.5^{\circ}$.

a strong contribution of an amorphous phase, which most probably originates from the PA starting powder as in agreement with its Raman analysis results.

\section{Electrical conductivity measurements}

Four-point probe measurements were performed on thin films on alumina membranes. The thickness, sheet resistance and conductivity values of the thin films are shown in Table 4. The sheet resistance and the corresponding conductivity values of the thin film prepared from the EG-IPA-90 min-TS dispersion are $7.3 \mathrm{k} \Omega /$ $\square$ and $2104 \mathrm{~S} / \mathrm{m}$, respectively. The thin films prepared from the IPA-based dispersions of the SEFG and PA precursors resulted in much higher sheets resistances $(\sim 807-858 \mathrm{k} \Omega / \square)$, leading to much lower conductivity values $(\sim 15 \mathrm{~S} / \mathrm{m})$ than that of the samples prepared from the EG powder. It should be noted that the NMP-based dispersions of these powders led to a slightly higher conductivity compared to IPA-based dispersions due to a better exfoliation (Table 4). These values are higher than those reported by Hernandez et al. [11] who obtained sheet resistance and conductivity values of $7.2 \mathrm{M} \Omega / \square$ and $5 \mathrm{~S} / \mathrm{m}$, respectively, for their thin graphene films prepared from dispersions of graphite in NMP. However, the authors improved the conductivity of the films to $6500 \mathrm{~S} / \mathrm{m}$ by annealing them in $\mathrm{Ar} / \mathrm{H}_{2}(90 \%-10 \%)$ atmosphere at $250{ }^{\circ} \mathrm{C}$ for $2 \mathrm{~h}$ [11]. The relatively low conductivity values of the graphene-based materials obtained from the SEFG and PA starting powders can be attributed to their small flake sizes. It is known that smaller flakes result in more junctions and consequently in lower conductivity due to effect of inter-flake junction resistances $[31,37]$. Moreover, the defects/disorders originating from the starting powder and the residual solvent that were revealed by the Raman and HRTEM analyses may act as scattering regions in these samples. Therefore, the thin films were annealed at $400{ }^{\circ} \mathrm{C}$ in $\mathrm{Ar} / \mathrm{H}_{2}$ (95:5\%) atmosphere for $4 \mathrm{~h}$, which resulted in a decrease in the resistance values (Table 4). Especially, the conductivity of the EGIPA-90 min-TS sample improved significantly reaching up to $19,200 \mathrm{~S} / \mathrm{m}$ (corresponding Rs: $0.8 \mathrm{k} \Omega / \square$ ), which is much better than many values reported in the literature $[6,11,12]$. However, the conductivity values of the graphene-based materials obtained from the SEFG and the PA starting powders are still too low for applications such as transparent electrodes, for which industry specifications require transparencies and sheet resistances better 


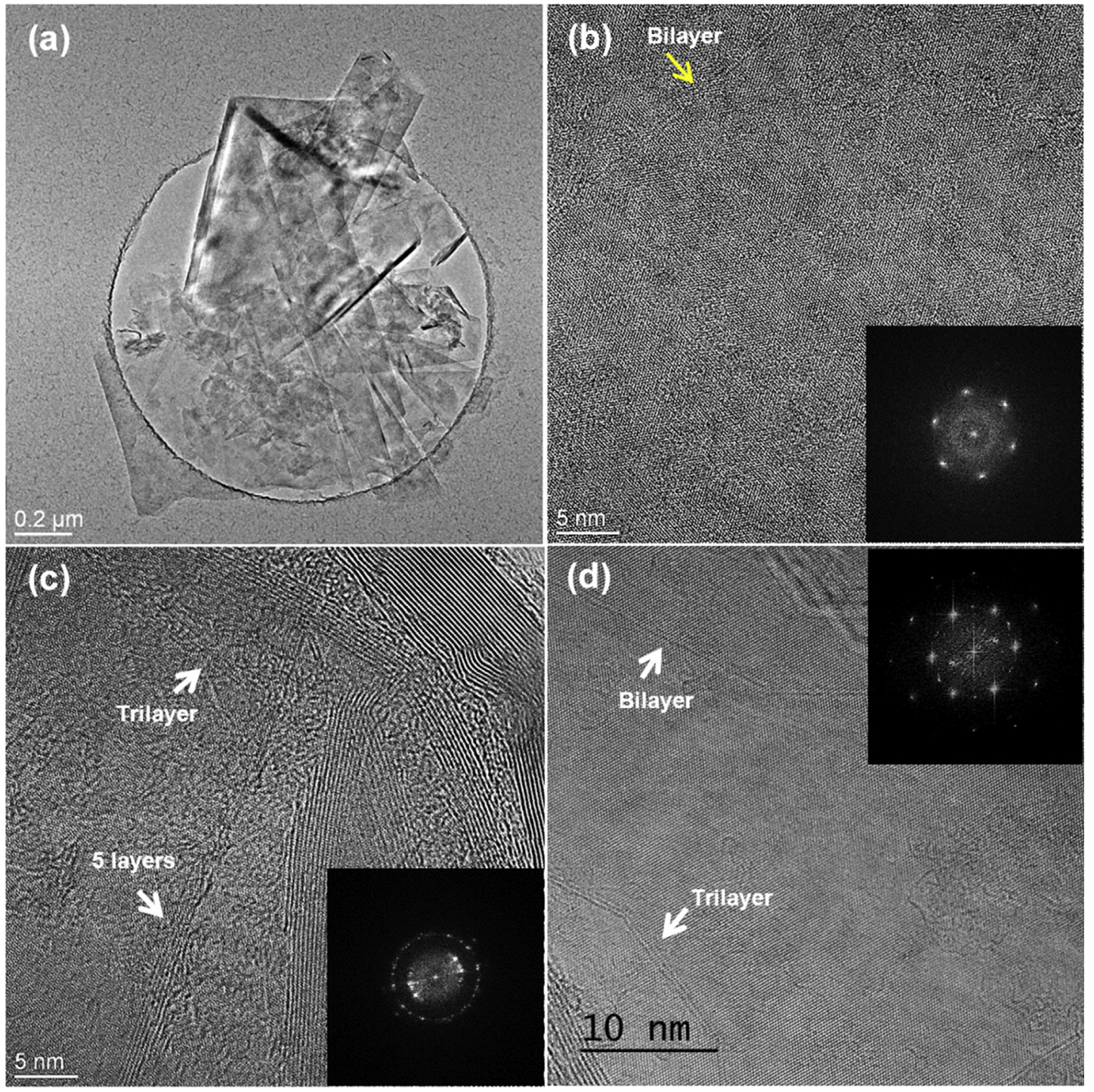

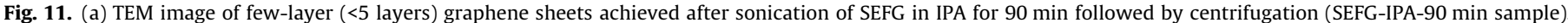

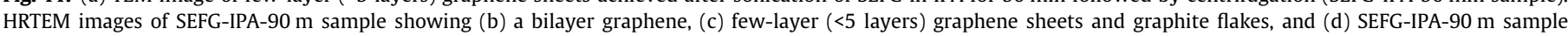
annealed at $2600{ }^{\circ} \mathrm{C}$ in $\mathrm{Ar}$ atmosphere for $4 \mathrm{~h}$ revealing bi-layer and three-layer graphene sheets. Insets show the FFTs of the corresponding HRTEM micrographs.

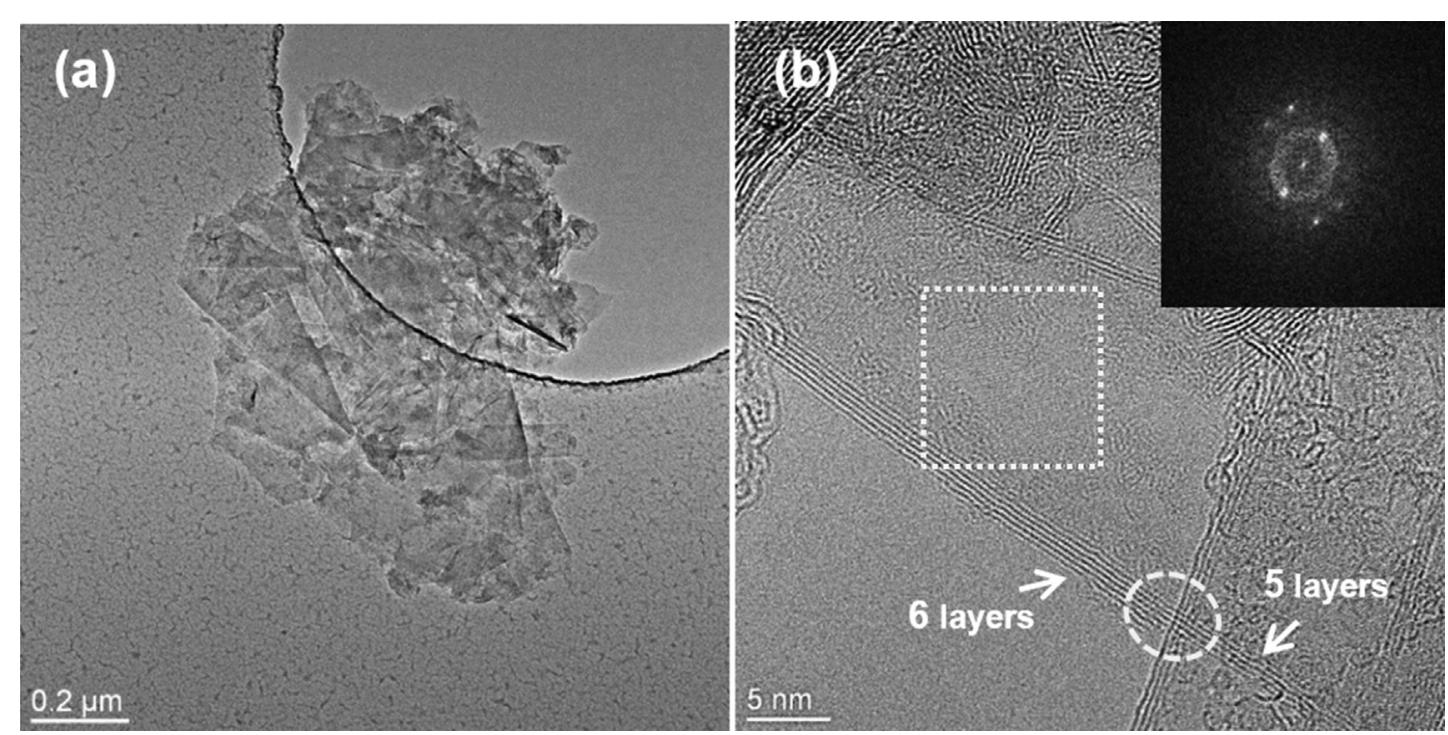

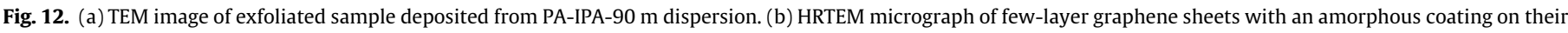
surface deposited from the PA-IPA-90 m dispersion. Inset of (b) shows the FFT image of the area indicated by a dashed rectangle. 
Table 4

Sheet resistance of graphene-based thin films measured by four-point probe technique and the corresponding conductivity values.

\begin{tabular}{|c|c|c|c|c|c|}
\hline & $\mathrm{t}(\mathrm{nm})$ & $\mathrm{R}_{\mathrm{s}}(\mathrm{k} \Omega / \square)$ & $\sigma_{\mathrm{el}}(\mathrm{S} / \mathrm{m})$ & $\mathrm{R}_{\mathrm{s}}\left(\right.$ annealed $\left.^{*}\right)(\mathrm{k} \Omega / \square)$ & $\sigma_{\mathrm{el}}($ annealed) $(\mathrm{S} / \mathrm{m})$ \\
\hline EG-IPA-90 min-TS & 65 & 7.3 & 2104 & 0.8 & 19200 \\
\hline SEFG-IPA-90min & 80 & 807.8 & 15 & 144.8 & 86 \\
\hline PA-IPA-90 min & 76 & 858.3 & 15 & 173.8 & 76 \\
\hline SEFG-NMP-90 min & 86 & 225.6 & 52 & 58.5 & 199 \\
\hline PA-NMP-90 min & 79 & 509.3 & 25 & - & - \\
\hline
\end{tabular}

$\mathrm{t}$ : thin film thickness.

$\mathrm{R}_{\mathrm{s}}$ : sheet resistance.

$\sigma_{\mathrm{el}}$ : electrical conductivity.

Annealing was performed at $400{ }^{\circ} \mathrm{C}$ in $\mathrm{Ar} / \mathrm{H}_{2}$ (95:5\%) atmosphere for $4 \mathrm{~h}$.

than $90 \%$ and less than $\sim 90 \Omega / \square$, respectively [37]. For these applications, the corresponding film conductivity that meets these requirements should be higher than $7 \times 10^{5} \mathrm{~S} / \mathrm{m}$ [37]. However, the electrical conductivity values achieved in the present study are enough to be incorporated into composites to improve the electrical properties of ceramic materials, as shown in our recent study [38].

\section{Conclusions}

Three different graphite-based materials (expandable graphite and two different nano-graphite powders) were investigated as starting powders for liquid phase exfoliation process in a low boiling point solvent, IPA, within relatively short sonication times $(<120 \mathrm{~min})$.

Good quality $\left(I_{\mathrm{D}} / I_{\mathrm{G}}<0.3\right)$ graphene-based dispersions with a relatively high concentration $(\sim 1.1 \mathrm{mg} / \mathrm{ml})$ have been successfully prepared in IPA within 90 min of bath sonication by utilizing a surface enhanced (SSA: $\sim 175 \mathrm{~m}^{2} / \mathrm{g}$ ) nano-graphite powder, which was derived from natural graphite. TEM and HRTEM analyses of this sample revealed mostly folded and scrolled few-layer graphene sheets (3-5 layers) entangled each other, as in agreement with its Raman analysis, as well as bilayer graphene sheets and few amount of thicker (>10 layers) graphite flakes. Sheet resistance measurements revealed a relatively high resistance $(807.8 \mathrm{k} \Omega / \square)$ and a low electrical conductivity value $(\sim 15 \mathrm{~S} / \mathrm{m})$ for the thin film ( $\sim 80 \mathrm{~nm}$ in thickness) prepared from this dispersion. Annealing of this thin film at $400{ }^{\circ} \mathrm{C}$ in $\mathrm{Ar} / \mathrm{H}_{2}$ atmosphere for $4 \mathrm{~h}$ decreased sheet resistance to $144.8 \mathrm{k} \Omega / \square$ and resulted in a conductivity value of $86 \mathrm{~S} / \mathrm{m}$. The relatively low electrical conductivity of this sample was attributed to small lateral sizes $\left(D_{50}: 403 \mathrm{~nm}\right)$ of the flakes, as well as the impurities/defects originating from the starting powder and the presence of residual solvent. Although this conductivity value is too low for transparent conductive electrode applications, using this starting material in LPE process was found to be advantageous for obtaining readily applicable high-concentration graphene-based materials for applications such as composites.

On the other hand, expanded graphite (prepared from expandable graphite) enabled the production of highly conductive ( $\sim 19,200 \mathrm{~S} / \mathrm{m}$ with the corresponding sheet resistance of $0.8 \mathrm{k} \Omega$ / $\square$ when annealed) and very good quality $\left(I_{D} / I_{G}<0.09\right)$ graphenebased materials with relatively large lateral sizes $(\sim \mathrm{a}$ few $\mu \mathrm{m})$ within 90 min of tip sonication followed by centrifugation. However, graphene-based material concentration of the prepared dispersion was relatively low $(\sim 0.06 \mathrm{mg} / \mathrm{ml})$ and it exhibited a polydisperse character.

\section{Acknowledgements}

The financial support for this study by Anadolu University Scientific Research Projects Commission (under the project numbers of 1110F155 and 1101F005) is gratefully acknowledged. The authors also thank to Dr. Pascal Puech (CEMES, Toulouse) for discussions on Raman analyses and to Lucien Datas for TEM analyses which were performed at R. Castaing characterization platform, UMS 3623.

\section{Appendix A. Supplementary data}

Supplementary data associated with this article can be found, in the online version, at http://dx.doi.org/10.1016/j.flatc.2016.12.002.

\section{References}

[1] W.C. Du, X.Q. Jiang, L.H. Zhu, From graphite to graphene: direct liquid-phase exfoliation of graphite to produce single- and few-layered pristine graphene, J. Mater. Chem. A 1 (2013) 10592-10606.

[2] A. Ciesielski, P. Samori, Graphene via sonication assisted liquid-phase exfoliation, Chem. Soc. Rev. 43 (2014) 381-398.

[3] J.N. Coleman, Liquid exfoliation of defect-free graphene, Acc. Chem. Res. 46 (2013) 14-22.

[4] L.Y. Niu, J.N. Coleman, H. Zhang, H. Shin, M. Chhowalla, Z.J. Zheng, Production of two-dimensional nanomaterials via liquid-based direct exfoliation, Small 12 (2016) 272-293.

[5] Y.L. Zhong, Z. Tian, G.P. Simon, D. Li, Scalable production of graphene via wet chemistry: progress and challenges, Mater. Today 18 (2015) 73-78.

[6] L. Guardia, M.J. Fernandez-Merino, J.I. Paredes, P. Solis-Fernandez, S. VillarRodil, A. Martinez-Alonso, J.M.D. Tascon, High-throughput production of pristine graphene in an aqueous dispersion assisted by non-ionic surfactants, Carbon 49 (2011) 1653-1662.

[7] S.M. Notley, Highly concentrated aqueous suspensions of graphene through ultrasonic exfoliation with continuous surfactant addition, Langmuir 28 (2012) $14110-14113$.

[8] D. Ager, V.A. Vasantha, R. Crombez, J. Texter, Aqueous graphene dispersionsoptical properties and stimuli-responsive phase transfer, ACS Nano 8 (2014) $11191-11205$.

[9] M. Ayan-Varela, J.I. Paredes, L. Guardia, S. Villar-Rodil, J.M. Munuera, M. DiazGonzalez, C. Fernandez-Sanchez, A. Martinez-Alonso, J.M.D. Tascon, Achieving extremely concentrated aqueous dispersions of graphene flakes and catalytically efficient graphene-metal nanoparticle hybrids with flavin mononucleotide as a high performance stabilizer, ACS Appl. Mater. Interfaces 7 (2015) 10293-10307.

[10] N. Behabtu, J.R. Lomeda, M.J. Green, A.L. Higginbotham, A. Sinitskii, D.V. Kosynkin, D. Tsentalovich, A.N.G. Parra-Vasquez, J. Schmidt, E. Kesselman, Y. Cohen, Y. Talmon, J.M. Tour, M. Pasquali, Spontaneous high-concentration dispersions and liquid crystals of graphene, Nat. Nano 5 (2010) 406-411.

[11] Y. Hernandez, V. Nicolosi, M. Lotya, F.M. Blighe, Z.Y. Sun, S. De, I.T. McGovern, B. Holland, M. Byrne, Y.K. Gun'ko, J.J. Boland, P. Niraj, G. Duesberg, S. Krishnamurthy, R. Goodhue, J. Hutchison, V. Scardaci, A.C. Ferrari, J.N. Coleman, High-yield production of graphene by liquid-phase exfoliation of graphite, Nat. Nanotechnol. 3 (2008) 563-568.

[12] U. Khan, A. O'Neill, M. Lotya, S. De, J.N. Coleman, High-concentration solvent exfoliation of graphene, Small 6 (2010) 864-871.

[13] U. Khan, H. Porwal, A. O’Neill, K. Nawaz, P. May, J.N. Coleman, Solventexfoliated graphene at extremely high concentration, Langmuir 27 (2011) 9077-9082.

[14] A. Catheline, C. Valles, C. Drummond, L. Ortolani, V. Morandi, M. Marcaccio, M. Iurlo, F. Paolucci, A. Penicaud, Graphene solutions, Chem. Commun. 47 (2011) 5470-5472.

[15] A. O'Neill, U. Khan, P.N. Nirmalraj, J. Boland, J.N. Coleman, Graphene dispersion and exfoliation in low boiling point solvents, J. Phys. Chem. C 115 (2011) 5422-5428.

[16] S.R. Dhakate, N. Chauhan, S. Sharma, J. Tawale, S. Singh, P.D. Sahare, R.B. Mathur, An approach to produce single and double layer graphene from reexfoliation of expanded graphite, Carbon 49 (2011) 1946-1954.

[17] N.V. Kozhemyakina, S. Eigler, R.E. Dinnebier, A. Inayat, W. Schwieger, A. Hirsch, Effect of the structure and morphology of natural, synthetic and postprocessed graphites on their dispersibility and electronic properties, Fullerness Nanotubes Carbon Nanostruct 21 (2013) 804-823. 
[18] J.D. Ingle, S.R. Crouch, Spectrochemical Analysis, Prentice Hall, Englewood Cliffs, NJ, 1988.

[19] A. Yu, C.-C.L. Su, I. Roes, B. Fan, R.C. Haddon, Gram-scale preparation of surfactant-free, carboxylic acid groups functionalized, individual single-walled carbon nanotubes in aqueous solution, Langmuir 26 (2009) 1221-1225.

[20] C.L. Chiang, S.W. Hsu, Synthesis, characterization and thermal properties of novel epoxy/expandable graphite composites, Polym. Int. 59 (2010) 119-126.

[21] G.X. Wang, J. Yang, J. Park, X.L. Gou, B. Wang, H. Liu, J. Yao, Facile synthesis and characterization of graphene nanosheets, J. Phys. Chem. C 112 (2008) 81928195.

[22] W.W. Liu, J.N. Wang, X.X. Wang, Charging of unfunctionalized graphene in organic solvents, Nanoscale 4 (2012) 425-428.

[23] M. Lotya, P.J. King, U. Khan, S. De, J.N. Coleman, High-concentration, surfactant-stabilized graphene dispersions, Acs Nano 4 (2010) 3155-3162.

[24] Zeta Potential of Colloids in Water and Waste Water, ASTM Standard D, American Society for Testing and Materials, 1985, pp. 4187-1482.

[25] A.C. Ferrari, Raman spectroscopy of graphene and graphite: disorder, electronphonon coupling, doping and nonadiabatic effects, Solid State Commun. 143 (2007) 47-57.

[26] M.S. Dresselhaus, A. Jorio, M. Hofmann, G. Dresselhaus, R. Saito, Perspectives on carbon nanotubes and graphene Raman spectroscopy, Nano Lett. 10 (2010) $751-758$.

[27] A.C. Ferrari, J.C. Meyer, V. Scardaci, C. Casiraghi, M. Lazzeri, F. Mauri, S. Piscanec, D. Jiang, K.S. Novoselov, S. Roth, A.K. Geim, Raman spectrum of graphene and graphene layers, Phys. Rev. Lett. 97 (2006).

[28] L.M. Malard, M.A. Pimenta, G. Dresselhaus, M.S. Dresselhaus, Raman spectroscopy in graphene, Phys. Rep. 473 (2009) 51-87.

[29] W.T. Gu, W. Zhang, X.M. Li, H.W. Zhu, J.Q. Wei, Z. Li, Q.K. Shu, C. Wang, K.L. Wang, W.C. Shen, F.Y. Kang, D.H. Wu, Graphene sheets from worm-like exfoliated graphite, J. Mater. Chem. 19 (2009) 3367-3369.
[30] I.M. Afanasov, O.N. Shornikova, D.A. Kirilenko, I.I. Vlasov, L. Zhang, J. Verbeeck, V.V. Avdeev, G. Tendeloo, Graphite structural transformations during intercalation by HNO3 and exfoliation, Carbon 48 (2010) 1862-1865.

[31] U. Khan, A. O’Neill, H. Porwal, P. May, K. Nawaz, J.N. Coleman, Size selection of dispersed, exfoliated graphene flakes by controlled centrifugation, Carbon 50 (2012) 470-475.

[32] M. Yi, Z.G. Shen, S.L. Ma, X.J. Zhang, A mixed-solvent strategy for facile and green preparation of graphene by liquid-phase exfoliation of graphite, J. Nanopart. Res. 14 (2012).

[33] R. Zan, Microscopy and spectroscopy of graphene: atomic scale structure and interaction with foreign atom species. vol. (Ph.D. thesis), School of Physics and Astronomy, University of Manchester, 2012.

[34] R. Zan, U. Bangert, Q. Ramasse, K.S. Novoselov, Imaging of Bernal stacked and misoriented graphene and boron nitride: experiment and simulation, J. Microsc.-Oxford 244 (2011) 152-158.

[35] G.X. Wang, X.P. Shen, J. Yao, J. Park, Graphene nanosheets for enhanced lithium storage in lithium ion batteries, Carbon 47 (2009) 2049-2053.

[36] S. Park, H.C. Floresca, Y. Suh, M.J. Kim, Electron microscopy analyses of natura and highly oriented pyrolytic graphites and the mechanically exfoliated graphenes produced from them, Carbon 48 (2010) 797-804.

[37] P.E. Lyons, S. De, F. Blighe, V. Nicolosi, L.F.C. Pereira, M.S. Ferreira, J.N. Coleman, The relationship between network morphology and conductivity in nanotube films, J. Appl. Phys. 104 (2008).

[38] Y. Celik, A. Celik, E. Flahaut, E. Suvaci, Anisotropic mechanical and functiona properties of graphene-based alumina matrix nanocomposites, J. Eur. Ceram. Soc. 36 (2016) 2075-2086. 